CHAPITRE 2

\title{
Écosystèmes aquatiques
}

HENRI DÉCAMPS 



\section{Introduction}

Depuis plusieurs décennies, nos besoins en eau s'accroissent et se diversifient - une tendance appelée à se poursuivre dans les décennies à venir. Pour être satisfaits, ces besoins exigent des milieux aquatiques en " bon état ». D'où l'objectif fixé par la directive cadre européenne sur l'eau : atteindre en 2015 un état ne s'écartant que légèrement $d$ 'une situation de référence - celle de milieux faiblement modifiés par les activités humaines ${ }^{1}$.

Cependant, les eaux continentales correspondent à des systèmes instables, susceptibles de passer d'un état à un autre, selon des trajectoires qui, ellesmêmes, évoluent en fonction de tendances à long terme - climatiques, hydrologiques, géomorphologiques (encadré 2.1). Les interactions entre les organismes vivants ajoutent à ces tendances des dynamiques à plus court terme, cycliques ou chaotiques, au gré d'assemblages infiniment variables dans l'espace et dans le temps. Tout « état » $d$ 'un système aquatique s'inscrit ainsi dans un ensemble de "régimes dynamiques », secoués de ruptures soudaines, rarement prévisibles.

Dans ces conditions, comment assurer un bon état des eaux continentales et, par suite, satisfaire les besoins en une eau de plus en plus rare et précieuse? Comment tendre vers ce bon état partout, dans la diversité des régions et des territoires? L'écologie propose une réponse originale à ces questions, en fixant un objectif et en présentant une méthode. L'objectif est de rendre les écosystèmes aquatiques plus résilients, $c^{\prime}$ est-à-dire plus aptes à se régénérer après perturbations, que ces dernières soient naturelles ou provoquées. La méthode est de gérer cette résilience (Folke et al., 2002). La résilience est en effet nécessaire pour utiliser les biens et les services de systèmes soumis, entre autres, à des crues et à des sécheresses de plus en plus probables et aiguës. Quant à la gestion, elle exige de mieux comprendre comment évoluent les interactions entre les milieux terrestres et aquatiques, à diverses échelles $d^{\prime}$ espace et de temps, sous l'effet de mécanismes multiples et interdépendants ${ }^{2}$.

Ce chapitre part de l'idée que les organismes vivants témoignent des états successifs des eaux continentales, et représentent un " capital nature " d'une valeur à bien des égards inestimable $(\S 1)$. Préserver ce capital conduit à retenir la notion de résilience comme clé $d^{\prime}$ un développement durable des écosystèmes

\footnotetext{
${ }^{1}$ La directive cadre définit le "bon état écologique " des eaux superficielles en référence à un état peu perturbé par les activités humaines pour ce qui est de la biologie du milieu, le « bon état chimique » des eaux superficielles et souterraines par rapport à des normes européennes sur l'écotoxicité et la toxicité pour l'homme, et le "bon état quantitatif» des eaux souterraines par rapport à un équilibre entre captage de la ressource en eau et renouvellement naturel de la nappe phréatique (Commission européenne).

${ }^{2}$ Voir aussi le «Millenium Assessment Report, Ecosystems and Human Well-being. Synthesis» (2005). Island Press, Washington, DC. www.maweb.org
} 
aquatiques (§ 2), à gérer cette résilience dans un environnement toujours fluctuant et incertain $(\S 3)$, et à prescrire des traitements capables d'assurer la santé écologique des eaux continentales ( $(4)$. Ces questions amènent à prendre conscience d'un devoir : celui d'une vision planétaire de "socio-écosystèmes » indissolublement liés aux eaux continentales (§5).

\section{Encadré 2.1}

\section{Un siècle de limnologie}

\section{Henri Décamps, Académie des sciences}

Qualifiée d'océanographie des lacs par son fondateur, le suisse FrançoisAlphonse Forel, la limnologie est née en 1901, avec la publication d'une monographie limnologique sur le Léman. Elle est ensuite devenue la science des eaux continentales, en s'intéressant aux relations qu'entretiennent les organismes aquatiques et riverains entre eux et avec leurs milieux - lacustres ou lotiques, superficiels ou souterrains, doux ou saumâtres.

La limnologie moderne est donc l'héritière d'un siècle d'histoire. Elle s'est particulièrement développée entre les deux grandes guerres mondiales, en Europe, en Amérique du Nord et au Japon. Parmi les figures marquantes de la discipline, l'Allemand August Thienemann et le Suédois Einar Nauman ${ }^{3}$ animèrent pendant une trentaine $d^{\prime}$ années, entre autres recherches, un vaste travail de classification des lacs de la planète, tandis qu'en Amérique du Nord, Edward Birge et Chancey Juday s'attachaient à replacer les lacs et leurs différents types le long de gradients environnementaux. Cette phase principalement descriptive prit fin avec G. Evelyn Hutchinson, auteur d'un monumental Traité de Limnologie et instigateur d'une bonne part des recherches menées de 1945 à 1980 sur l'écologie des eaux continentales.

De 1945 à 1960, les avancées les plus marquantes ont porté sur : le métabolisme des rivières, l'influence des bassins versants sur le fonctionnement écologique et biogéochimique des cours d'eau, la modélisation prédictive de l'eutrophisation des lacs et leur réhabilitation, la manipulation in situ des réseaux trophiques. Ces avancées, souvent réalisées par de petites équipes dans un souci d'application, ont stimulé des recherches plus fondamentales sur les mécanismes écologiques sous-jacents. Elles ont aussi ouvert la voie, notamment sous I'influence du Programme biologique international entre

\footnotetext{
${ }^{3}$ Thienemann et Nauman fondèrent la Société internationale de limnologie théorique et appliquée en 1922, une société aujourd'hui forte d'environ 2800 adhérents répartis en plus de 80 pays, tenant congrès tous les quatre ans (sa branche française est l'Association française de limnologie, http://aflimno.free.fr).
} 
1960 et 1980, au développement de modèles quantitatifs de plus en plus réalistes. Les travaux menés en France, dans les différents organismes de recherche et dans les universités, ont largement contribué à ces avancées (Pourriot et Meybeck, 1995).

Les dernières décennies du $X X^{e}$ siècle ont vu s'estomper les limites entre limnologie fondamentale et appliquée. Surtout, elles se sont démarquées des décennies précédentes (Kalff, 2002) : d'un point de vue technique, les méthodes d'observations en continu, y compris par télédétection, ont permis de disposer de quantités considérables de données, en même temps que se développaient les capacités de modélisation et que s'affinaient les analyses permettant de détecter, par exemple, les produits contaminants ; d'un point de vue conceptuel, les écosystèmes aquatiques continentaux sont apparus comme des systèmes ouverts, largement influencés par les caractéristiques de leurs bassins versants, et les communautés aquatiques comme des systèmes en état de non-équilibre quasi permanent. Mais la structuration de ces communautés continue à diviser les tenants des approches par populations et par écosystèmes : les premiers privilégient les interactions entre espèces (prédation, compétition, parasitisme, symbiose), les seconds privilégient plutôt la disponibilité des ressources (nutriments, lumière).

Au-delà de ce débat, et quel que soit son intérêt, la limnologie est confrontée à deux défis majeurs en ce début de $\mathrm{XXI}^{\mathrm{e}}$ siècle. Le premier défi concerne la question des échelles d'espace et de temps - y compris la question des relations entre ces échelles - auxquelles il convient de se placer face aux changements de notre environnement (voir $\S 2.2$ et 3.1 ). Le second défi concerne l'articulation de la limnologie avec d'autres sciences, particulièrement celles de l'homme et de la société, pour imaginer des solutions aux problèmes posés par ces changements, une articulation qui renvoie à des notions telles que celle de socio-écosystème (voir $§ 2.3$ ). Répondre à ces défis suppose une organisation plus cohérente et plus interdisciplinaire des recherches conduites en France sur les eaux continentales, une organisation qu'il faudrait repenser, à un niveau européen, sans oublier le devoir d'une vision planétaire (voir $§ 5$ ).

\section{Les organismes vivants, témoins des états des eaux continentales}

Par leur présence, les assemblages d'organismes aquatiques témoignent des événements qui se sont déroulés dans les lacs et les rivières, ainsi que sur les terres de leurs bassins versants. Ces assemblages résultent en effet d'adaptations millénaires à des conditions climatiques, géologiques, chimiques et biologiques. 
Ce sont des indicateurs de l'état de santé des eaux continentales - un état de santé qui conditionne les usages des lacs et des rivières, qu'il s'agisse de produire de l'eau potable, de générer de l'électricité, de pêcher, de naviguer ou $d^{\prime}$ 'irriguer. Ces indicateurs biologiques doivent permettre de suivre les transformations, naturelles ou provoquées, de l'état des eaux continentales.

Cette première partie porte sur la notion d'état de santé des écosystèmes aquatiques, sur la surveillance de cet état et sur les perturbations susceptibles de le modifier. Elle évoque également la valeur du capital nature associé aux systèmes aquatiques.

\subsection{La santé des écosystèmes}

Les écosystèmes aquatiques sont transformés par les activités humaines. En certains cas, cette transformation peut aller jusqu'à la disparition pure et simple d'écosystèmes aussi bien établis que ceux de la mer d'Aral. La notion de santé des écosystèmes permet d'évaluer jusqu'où ces transformations restent acceptables. Comment définir cette notion? Par quelles méthodes la mesurer? Par quels indicateurs? Ces questions sont discutées au sein d'une Société internationale de santé des écosystèmes qui, depuis 1994, réunit des scientifiques de divers domaines, des sciences de la nature aux sciences de l'homme et de la société.

La notion de santé des écosystèmes est souvent associée à celle d'intégrité. Ainsi, en 1992, la conférence des Nations unies sur l'environnement et le développement faisait obligation aux États membres de "sauvegarder la santé et I'intégrité des écosystèmes de la planète ". Il importe toutefois de distinguer ces deux notions. Un écosystème en bonne santé assure le recyclage de l'énergie et des nutriments; il maintient ses fonctions essentielles de production, de consommation, et de décomposition; il s'adapte aux effets à long terme des perturbations naturelles. II est capable de « s'auto-renouveler 》 et, malgré les stress, de conserver sa résilience, sa diversité et sa productivité, attributs dont dépend une utilisation durable de ses services par la société. Pour sa part, la notion d'intégrité écologique renvoie à des états de référence qui, supposés indemnes $d^{\prime}$ interventions humaines significatives, fournissent une base d'évaluation des écosystèmes actuels. Bref, les deux notions se complètent : la santé évalue l'aptitude des écosystèmes à délivrer une gamme acceptable de services, l'intégrité procure un étalon de mesure pour cette évaluation.

Cette distinction se précise quand on replace la notion de santé écologique le long d'un gradient, celui de l'influence humaine sur les milieux aquatiques (figure 2.1). Le problème revient à identifier le seuil séparant les conditions de 
" bonne santé » des conditions de " mauvaise santé ». Où placer ce seuil ? Selon quels critères? Quand la dégradation de la santé du système devient-elle irréversible? Quand est-elle ressentie comme intolérable par le public?

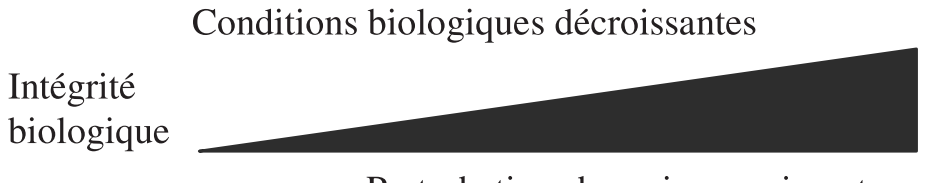

Dégradation sévère

Perturbations humaines croissantes

Bon état

écologique

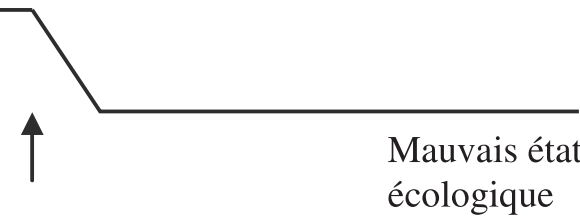

seuil

Figure 2.1

État de santé d'un écosystème aquatique (en bas) en fonction d'une influence humaine perturbatrice croissante (en haut). Les systèmes peu perturbés par les activités humaines conservent une certaine intégrité biologique. Avec l'augmentation des perturbations, la santé de l'écosystème s'altère soudainement au-delà d'un certain seuil (d'après Karr et Chu, 1999).

L'identification objective de ce seuil se heurte à la diversité des perceptions de la bonne santé des lacs et des rivières : celles des agriculteurs, des ingénieurs, des ménagères, des pêcheurs, des protecteurs de la nature diffèrent les unes des autres. Elle se heurte en outre à la diversité des méthodes utilisées par les scientifiques pour questionner la nature. On accepte généralement l'idée que : 1) les activités humaines ne doivent pas altérer la capacité $d^{\prime}$ 'une zone donnée à soutenir durablement les biens et les services fournis par cette zone, et que, 2) elles ne doivent pas non plus dégrader, par répercussion, $\mathrm{d}^{\prime}$ autres zones que celle considérée, ce qui implique une vision de l'état des eaux continentales élargie à l'ensemble des bassins versants. En fait, l'identification des seuils entre bonne et mauvaise santé écologique est $d^{\prime}$ autant moins subjective qu'elle s'appuie sur une surveillance biologique rigoureuse des écosystèmes aquatiques.

Comment définir un écosystème de référence? Souvent, aucun site n'y correspond vraiment, aucun ne peut exprimer à lui seul tous les attributs de l'intégrité écologique, ni ses états successifs. L'identification d'un écosystème de référence s'appuie donc sur de multiples sites et fait appel à des traits relevés antérieurement aux dommages subis, ou caractéristiques d'autres sites semblables moins dégradés. On tente ainsi de dégager les traits physicochimiques majeurs " naturels ", la périodicité des événements perturbateurs " normaux ", certains aspects 
de la biodiversité comme la composition spécifique et la structure des communautés. Tout ceci demande de l'expérience et du jugement, et ne va pas sans discussions, ni sans recherches.

\subsection{Surveillance biologique des eaux}

La surveillance biologique de l'état des écosystèmes conditionne leur protection, en eau douce comme ailleurs. Cette surveillance porte sur des indices dits " multimétriques » car ils s'appuient sur plusieurs attributs - ou métriques pour évaluer l'état d'un lac ou d'un secteur de rivière. Les bonnes métriques sont celles qui reflètent clairement les réponses biologiques aux activités humaines : elles varient de manière prévisible en fonction de ces activités; elles réagissent à un ensemble et non à un type particulier de stress; elles permettent de distinguer les changements provoqués par les activités humaines du «bruit de fond 》 des variations naturelles.

Le choix de ces métriques suppose une bonne connaissance de l'histoire naturelle des milieux aquatiques. Il s'agit en effet de disposer $d^{\prime}$ indices réellement liés à la vie aquatique et susceptibles d'orienter les gestionnaires vers de bonnes décisions, tout en étant compris par le public. Pour les cours d'eau, une dizaine de métriques ont été proposées, par exemple sur les poissons (tableau 2.1). D'autres ont aussi été développées, sur les macro-invertébrés, les algues et les végétaux vasculaires. Regroupées en indices, ces métriques permettent un

Nombre d'espèces natives

Nombre d'insectivores de fond à courant rapide

Nombre d'insectivores de pleine eau

Nombre d'insectivores de fond à courant lent

Nombre d'espèces intolérantes

Abondance relative des individus d'espèces tolérantes

Abondance relative d'omnivores

Abondance relative d'insectivores

Abondance relative de top carnivores

Abondance relative d'individus malades

Tableau 2.1

Exemple de métriques relatives aux poissons, testées et utilisées dans des indices multimétriques d'intégrité biologique. 
diagnostic des effets des activités humaines à divers niveaux d'échelles (individu, population, communauté, écosystème, paysage). Leur précision dépend des progrès de nos connaissances quant aux espèces présentes, aux états physiologiques, aux données démographiques, aux structures trophiques, au recyclage des nutriments et des flux d'énergie.

La surveillance biologique de l'état des eaux continentales s'insère donc dans une logique qui conduit à protéger la vie aquatique en référence à des conditions d'intégrité biologique (tableau 2.2). Dans cette logique, les sources d'altération concernent :

- le régime des débits (crues et étiages en décalage par rapport aux cycles de vie des organismes, prélèvements d'eau excessifs);

- la structure physique des habitats (recalibration des rivières, sédimentations accrues de frayères, diminution du bois mort immergé, destruction de la végétation riveraine);

- la qualité de l'eau (eaux plus chaudes, plus troubles, moins oxygénées, diversement contaminées);

- les sources d'énergie (apports de matière organique);

- les interactions biotiques (processus de compétition, de prédation, de mutualisme, de parasitisme).

La directive cadre européenne a incité à intensifier les efforts en vue d'évaluer la qualité biologique des cours d'eau, notamment en France avec l'amélioration des indices couramment utilisés sur les macro-invertébrés, les diatomées ou les poissons. À l'échelle européenne, le système AQEM (Aquatic Quality Evaluation Method), récemment mis au point, prend en compte une grande diversité de situations et s'appuie sur 9557 espèces de macro-invertébrés, dans une approche multisite et multimétrique (Hering et al., 2004). Ce système vise à classer les tronçons de cours d'eau en cinq classes de qualité, tout en identifiant les causes possibles de dégradation.

L'état écologique d'un cours d'eav évalué à partir des espèces est-il identique à celui évalué à partir des genres ou des familles? Autrement dit, l'indice de qualité attribué à un site change-t-il quand on extrapole des métriques calculées au niveau spécifique à d'autres niveaux taxinomiques? Les travaux récents conduisent à répondre oui à cette question : les évaluations se révèlent moins précises en passant du niveau spécifique aux niveaux taxinomiques plus élevés. D'où l'intérêt du choix des espèces comme indicateurs biologiques, et la nécessité de l'expertise dans le domaine de la systématique. 


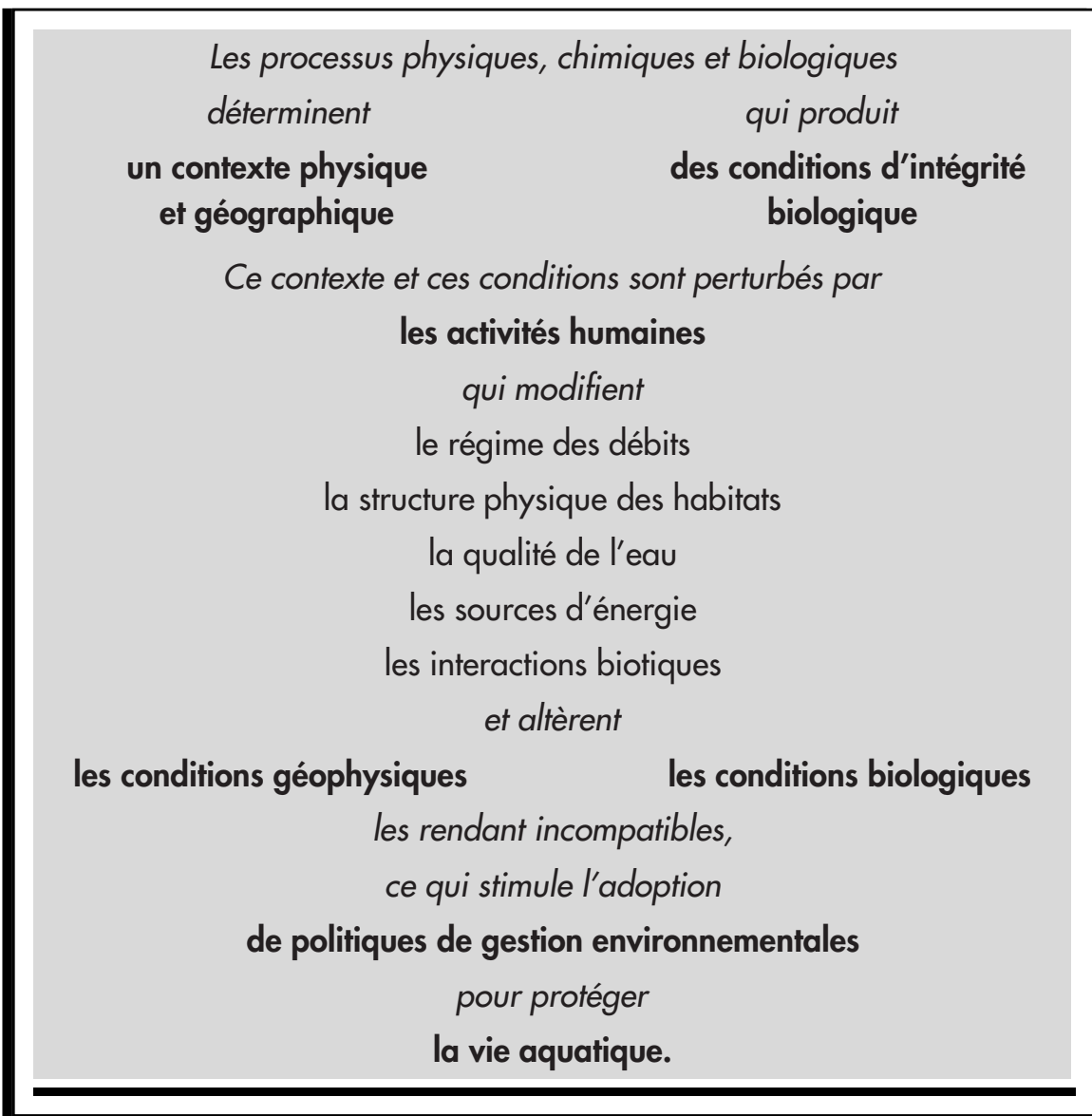

Tableau 2.2

Logique d'une surveillance biologique des eaux continentales tenant compte des relations entre les différentes variables en cause (d'après Karr et Chu, 1999).

\subsection{Perturbations naturelles et provoquées}

Le nombre de grandes crues risque d'augmenter avec le changement climatique et l'avènement probable de pluies hivernales extrêmes, par exemple en Europe du Nord et en certaines régions de l'Asie du Sud (Palmer et Räisänen, 2002). Les habitants du Bangladesh, dans la plaine inondable de trois grands fleuves, vivent déjà une situation paroxystique : des millions d'entre eux ont été déplacés par des crues désastreuses, auxquelles s'ajoutent des périodes de sécheresse et la recrudescence de maladies liées à l'eau. 
II est difficile de distinguer la part naturelle de ces perturbations de celle provoquée par les activités humaines. En principe, les perturbations naturelles renouvellent les écosystèmes : elles sont indispensables au maintien de leur intégrité, au point que l'absence de perturbation naturelle est elle-même considérée comme une perturbation, par exemple dans le cas de la régularisation du régime des débits d'une rivière. Peut-être peut-on souligner que les perturbations provoquées par les activités humaines diffèrent de celles susceptibles de se produire dans les conditions naturelles quant à leur type, leur fréquence, leur expansion ou leur degré de prévisibilité : beaucoup de perturbations subies par les écosystèmes aquatiques s'écartent aujourd'hui des normes établies sur l'histoire du dernier millénaire (Naiman et al., 2005).

La notion d'événement extrême s'avère aussi difficile à définir. D'abord, ces événements sont divers : certains, relativement soudains et courts, agissent par pulsations (une crue), d'autres apparaissent aussi soudainement mais se prolongent dans le temps, exerçant une pression continue (une sédimentation consécutive à un effondrement de terrain), d'autres encore voient leurs effets s'aggraver avec le temps (une sécheresse). Ensuite, les espèces peuvent elles-mêmes réagir différemment à une même perturbation. Enfin, entre en jeu une perception sociale, variable dans l'espace et dans le temps, de tout événement perturbateur.

Il est encore plus délicat de prévoir les réponses des écosystèmes aux perturbations anthropiques. Ces réponses n'apparaissent souvent qu'après un temps de latence plus ou moins long, plusieurs années s'avérant nécessaires pour observer, par exemple, les effets d'un dépôt acide, d'une espèce introduite ou d'une pollution des eaux souterraines (encadré 2.2). Aux questions posées par l'existence de ces temps de latence s'ajoutent celles liées à l'accumulation des impacts : les effets s'ajoutent-ils les uns aux autres de façon linéaire, s'amplifientils, introduisent-ils des seuils plus sévères? Au total, ces temps de latence et ces effets cumulatifs compliquent singulièrement la prévision des trajectoires à venir des écosystèmes aquatiques, $d^{\prime}$ 'autant plus que ces derniers intègrent les événements, des plus habituels aux plus extrêmes, qui se déroulent sur l'ensemble de leurs bassins versants.

Comment accompagner les effets du changement climatique sur les écosystèmes aquatiques continentaux? Cette tâche s'avère difficile car ces systèmes sont très sensibles aux élévations de température, dans leur fonctionnement comme dans leur composition spécifique. Et leurs espèces sont particulièrement vulnérables aux modifications des régimes d'écoulement. De plus, les effets du changement climatique s'ajoutent, par exemple, aux dégradations de la qualité des eaux. Il s'avère donc nécessaire d'adapter les méthodes de suivi de l'état des eaux aux conséquences du changement climatique, tout en minimisant les prélèvements d'eau souterraine et les effets du fonctionnement des barrages, en réduisant les charges en nutriments, et en maintenant des boisements riverains. 


\title{
Encadré 2.2
}

\section{Échanges à l'interface entre eaux de surface et souterraines}

\author{
Janine Gibert, université Claude Bernard Lyon 1
}

Depuis une vingtaine d'années l'interface eaux de surface/eaux souterraines a fait l'objet de diverses études écologiques (Gibert et al., 1997). Il a été démontré que les échanges (physiques, chimiques et biologiques) au niveau de la zone d'interface jouent un rôle majeur dans la régulation des flux de nutriments circulant dans les cours d'eau et de la biodiversité globale des systèmes aquatiques. Deux exemples permettent $d^{\prime}$ illustrer ces recherches.

\section{Effets des aménagements des grands fleuves}

L'interface entre les eaux de surface et les eaux souterraines correspond à une zone d'échanges privilégiés. Les communautés interstitielles, assemblages faunistiques à l'interface entre les eaux de surface et les eaux souterraines, jouent un rôle majeur dans la dynamique de ces échanges. En milieu fluvial, elles apparaissent comme de véritables « descripteurs du fonctionnement », intégrant les modifications des conditions de milieu sous l'effet des aménagements. Par exemple, les recherches effectuées sur le Haut-Rhône ont montré que la diversité des traits biologiques des espèces, en particulier les catégories trophiques, répondaient aux modifications de la qualité et de la quantité des nutriments reçus par le milieu interstitiel; de même, les modes de déplacement des espèces traduisent les possibilités $d^{\prime}$ accès aux annexes fluviales, et les différentes classes de taille rendent compte des modifications des interstices et donc de l'habitabilité du milieu.

\section{Protection des eaux souterraines en milieu urbain}

Souvent placés dans les plaines alluviales des grands fleuves, les ensembles urbains concentrent les flux (eau, polluants et nutriments) vers les nappes phréatiques utilisées par ailleurs comme source d'eau potable. D'où l'intérêt du rôle de filtre naturel joué par la zone non saturée des aquifères dont les premiers centimètres filtrent les métaux lourds et les hydrocarbures. Des organismes comme les oligochètes, en multipliant leurs galeries, augmentent les échanges entre l'eau libre et les sédiments, et stimulent les processus de dégradation de la matière organique. L'épaisseur de la zone non saturée apparaît ainsi comme un paramètre prépondérant qui module les effets de l'infiltration des eaux pluviales et permet une meilleure protection des eaux souterraines. 
Les échanges entre les eaux superficielles et souterraines, notamment à l'échelle européenne, font l'objet de divers programmes d'études. Ces programmes tentent $d^{\prime}$ estimer la biodiversité aquatique souterraine, et son rôle dans le fonctionnement, tant des écosystèmes aquatiques souterrains que de leurs interfaces avec les milieux de surface. Leur objectif est d'élaborer des plans d'action visant à intégrer la biodiversité souterraine dans les politiques de conservation et de gestion de l'environnement. C'est le cas par exemple du programme européen PASCALIS (Protocol for the ASsessment and Conservation of Aquatic Life In the Subsurface) ${ }^{4}$ qui définit des recommandations à intégrer : (1) dans la directive cadre sur l'eau (considération de la ressource en eau souterraine comme un milieu vivant, introduction pour les eaux souterraines du statut de " bon état écologique », proposition d'indicateurs d'échanges entre eaux de surface et eaux souterraines) et (2) dans la directive habitat (protocoles pour définir des espèces et des sites prioritaires pour la conservation à l'échelle de l'Europe).

\subsection{Le « capital nature »}

Les écosystèmes aquatiques représentent un capital nature, source de bénéfices pour la société. Ils sont susceptibles de rendre des services dont l'évaluation est difficile car ils ne sont pas pris en compte dans les analyses économiques classiques (tableau 2.3).

En 1997, Costanza et al. évaluèrent le « prix » des services écologiques pour l'ensemble de la planète à une valeur à peu près égale au PNB global de l'époque, $18 \%$ de cette valeur revenant aux eaux continentales. Cette évaluation déclencha une controverse, dans la mesure où les extrapolations impliquées ne prenaient pas en compte la façon dont varie la demande des services considérés et où certaines méthodes utilisées pour évaluer ces services sont contestables. En outre, il n'était pas tenu compte des bénéfices liés à certaines transformations des milieux naturels. Un écosystème peut en effet délivrer des services substantiels - éventuellement différents - après transformation intentionnelle. II importe alors d'identifier les différences entre les flux de bénéfices existant avant et après transformation. En leur temps, le drainage de certains marais a largement bénéficié à l'agriculture comme à la santé humaine.

II existe pourtant des raisons économiques pour conserver notre capital nature (Balmford et al., 2002). Et la dégradation actuelle des écosystèmes aquatiques pose des problèmes de survie en de nombreuses régions de la planète.

\footnotetext{
${ }^{4} \mathrm{http}: / /$ www.pascalis-project.com
} 
Alimentation en eau agricole, industrielle et domestique.

Source de nourriture, notamment poissons.

Purification de l'eau et élimination des déchets par les zones humides.

Atténuation des effets des crues par rétention des eaux sur les bassins versants et dans les plaines inondables.

Atténuation des effets des sécheresses par recharge des aquifères à partir des eaux de surface.

Habitats variés, abris et lieux de reproduction de poissons, oiseaux et autres espèces sauvages.

Renouvellement de la fertilité des sols proches des systèmes d'eau courante. Apports de nutriments aux deltas, estuaires et milieux marins côtiers.

Maintien des gradients de salinité des deltas et zones marines côtières, source de richesse biologique et de productivité.

Contribution à la beauté des paysages, à la qualité de la vie, ainsi qu'à des valeurs culturelles et spirituelles.

Sources de loisirs : pêche, nage, canotage, observation de la nature, parcours riverains...

Conservation de la biodiversité.

\section{Tableau 2.3}

Services vitaux rendus par les écosystèmes aquatiques continentaux (d'après Postel et Richter, 2003).

En ces circonstances, l'association de l'écologie et de l'économie doit livrer les bases nécessaires aux politiques d'amélioration de l'état des eaux continentales. Elle doit aussi permettre de mieux comprendre les équilibres entre les différents services rendus par les écosystèmes aquatiques, tout en précisant en quoi l'utilisation trop poussée d'un service donné entrave celle d'autres services et affecte des fonctions écologiques vitales. À cet égard, le Rapport de synthèse sur l'évaluation des écosystèmes pour le Millénaire cite la production de ressources halieutiques et la production d'eau douce comme deux services aujourd'hui rendus à un niveau bien inférieur aux besoins actuels, sans parler des besoins futurs ${ }^{5}$.

\section{La résilience des socio-écosystèmes aquatiques}

Les écosystèmes en bonne santé s'adaptent aux changements de leur environnement et se réorganisent après perturbation sans perdre leurs fonctions vitales.

${ }^{5}$ http://www.maweb.org 
Cette aptitude à se régénérer correspond à la résilience. Cependant, tout écosystème peut perdre cette aptitude et, suite à de fortes perturbations, s'engager vers de nouveaux états, souvent jugés indésirables. Un défi majeur pour l'aménagement des eaux continentales est de comprendre les causes de ces alternances d'états.

\subsection{Alternances d'états}

L'état d'un écosystème peut fluctuer pendant un certain temps autour de valeurs moyennes, selon des tendances relativement stables et prévisibles. Un régime de croisière s'établit qui, toutefois, peut s'interrompre soudainement pour faire place à un nouveau régime, à la surprise des observateurs. De telles alternances de régimes ne sont pas rares et l'un des exemples les plus connus est celui de la crise affectant les écosystèmes coralliens (Bellwood et al., 2004). À aucun moment, la bonne connaissance du fonctionnement de ces écosystèmes ne permettait de prévoir leur encroûtement par des algues, et un certain recul était nécessaire pour comprendre la suite des événements à l'origine de la crise : l'exploitation plus poussée des terres a grossi les apports de nutriments au milieu aquatique, amplifiant la croissance des algues; cette croissance a d'abord été masquée par l'action d'abondantes populations de poissons herbivores et, suite au déclin de ces populations intensément pêchées, par la prolifération d'une espèce d'oursin elle-même herbivore, cette prolifération a favorisé le développement d'un agent pathogène, la disparition des oursins et, désormais en l'absence de tout contrôle, le développement d'algues encroûtantes.

Des exemples semblables existent en milieu lacustre, notamment avec la perte de transparence en certains lacs peu profonds. Sous l'effet des apports en nutriments provenant du bassin versant, ces lacs - écosystèmes aux eaux claires abritant de nombreuses plantes submergées - voient leur concentration en nutriments augmenter, sans paraître au début affecter la clarté des eaux. Mais au-delà d'un sevil critique, la prolifération des algues du phytoplancton installe une forte turbidité qui cause la disparition des plantes submergées et des animaux associés à ces plantes. Or, quand on tente de ramener ces milieux à leur état d'origine, la réduction de la concentration en nutriments s'avère souvent insuffisante pour restaurer la clarté des eaux : cette réduction ne devient effective qu'à des concentrations nettement inférieures à celles qui marquaient le début de la disparition des végétaux submergés (figure 2.2). Le retour à une eau claire implique en effet une réduction des nutriments dans la colonne d'eau, mais aussi un arrêt des remises en suspension des sédiments du fond et le redéploiement d'un zooplancton s'alimentant à partir de phytoplancton. Ce retour peut être contrarié par la présence des poissons qui, en l'absence de plantes submergées, exercent une forte prédation sur le zooplancton, tout en remettant fréquemment en suspension les sédiments du fond. 


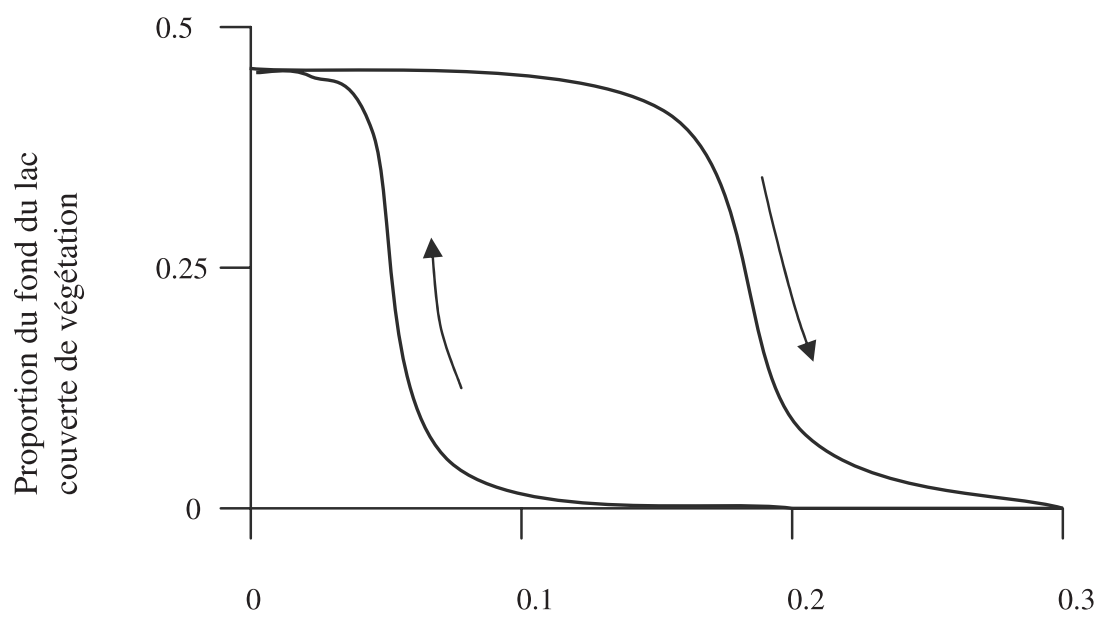

Concentration en phosphore total (mg/l).

\section{Figure 2.2}

Réponse du couvert végétal du fond du lac peu profond à une augmentation, puis à une diminution de la concentration en phosphore (d'après Scheffer et al., 2001).

De telles crises peuvent survenir suite à une légère transformation des conditions extérieures, lorsque le dépassement d'un seuil critique provoque le basculement d'un état à un autre. Ainsi, un écosystème situé de par son état sur l'une des branches continues de la courbe de la figure 2.3 ne passera pas graduellement à un état qui le placera sur l'autre branche continue de la courbe : il y passera soudainement au niveau d'un seuil. Premièrement, avant d'atteindre ce seuil, un système placé sur la branche supérieure d'un état stable désirable n'évolue que faiblement, rien ne laissant prévoir à l'avance son basculement. Deuxièmement, on ne ramène pas un système d'un état indésirable à un état désirable par un simple retour au point de basculement F2, mais par un retour au point $\mathrm{Fl}$. Ce cycle d'hystérésis peut varier fortement d'un système à l'autre, voire au sein d'un même système : des lacs peu profonds peuvent ainsi présenter une hystérésis prononcée en réponse à un apport de nutriments, tandis que des lacs profonds réagiront de manière plus graduelle, certains présentant même des différences entre leurs zones superficielles et profondes.

Un changement environnemental progressif peut donc modifier le « domaine d'attraction " de l'état d'un écosystème, et augmenter le risque de basculement vers un autre état sous l'effet de perturbations même faibles. Ces basculements sont difficiles à gérer : d'une part, il n'existe généralement pas de signes précoces du changement d'un état désirable vers un état indésirable; d'autre part, une fois ce changement réalisé, les restaurations s'avèrent longues et coûteuses 


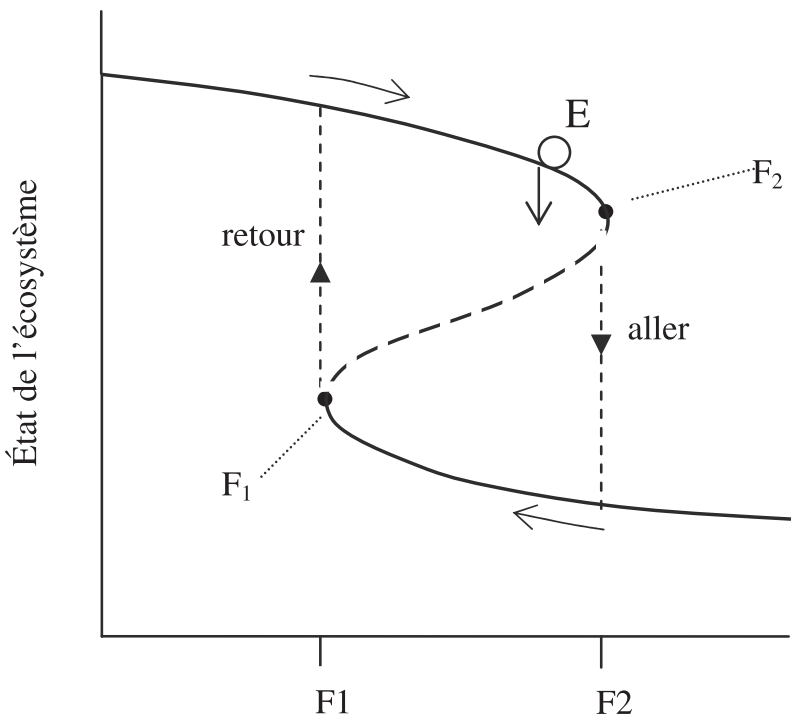

Conditions

\section{Figure 2.3}

Aller et retour entre deux états stables alternatifs. Un léger déplacement des conditions d'environnement vers la droite conduit l'écosystème placé en E sur la partie supérieure de la courbe à dépasser le point de bifurcation F2 et à basculer vers l'autre état stable dans la partie inférieure de la courbe (aller). Un retour à l'état stable du haut demande à revenir aux conditions d'environnement qui prévalaient à la hauteur du point de bifurcation Fl (d'après Scheffer et al., 2001).

à cause de la présence d'hystérésis. Les efforts portent donc souvent sur la prévention des perturbations et non sur le problème sous-jacent de la perte de résilience. Or, les perturbations qui déclenchent les changements d'état - tempêtes, sécheresses, épidémies - sont difficiles à prévoir et à modifier, contrairement aux variables qui contrôlent la résilience, par exemple le mode d'utilisation des terres, le stockage des nutriments, la connectance des chaînes trophiques. La gestion de ces variables - et donc de la résilience - est probablement la meilleure façon de gérer les états désirés d'un écosystème soumis au changement des conditions de l'environnement.

\subsection{Cycles adaptatifs et emboîtements d'échelles}

Les systèmes naturels et humains interagissent au cours de transformations cycliques, avec des phases successives de croissance, de conservation, de destruction et de renouvellement (figure 2.4). Ces cycles sont adaptatifs, c'est-à-dire susceptibles d'innover face aux crises. Et cette capacité d'innover dépend de trois propriétés : 


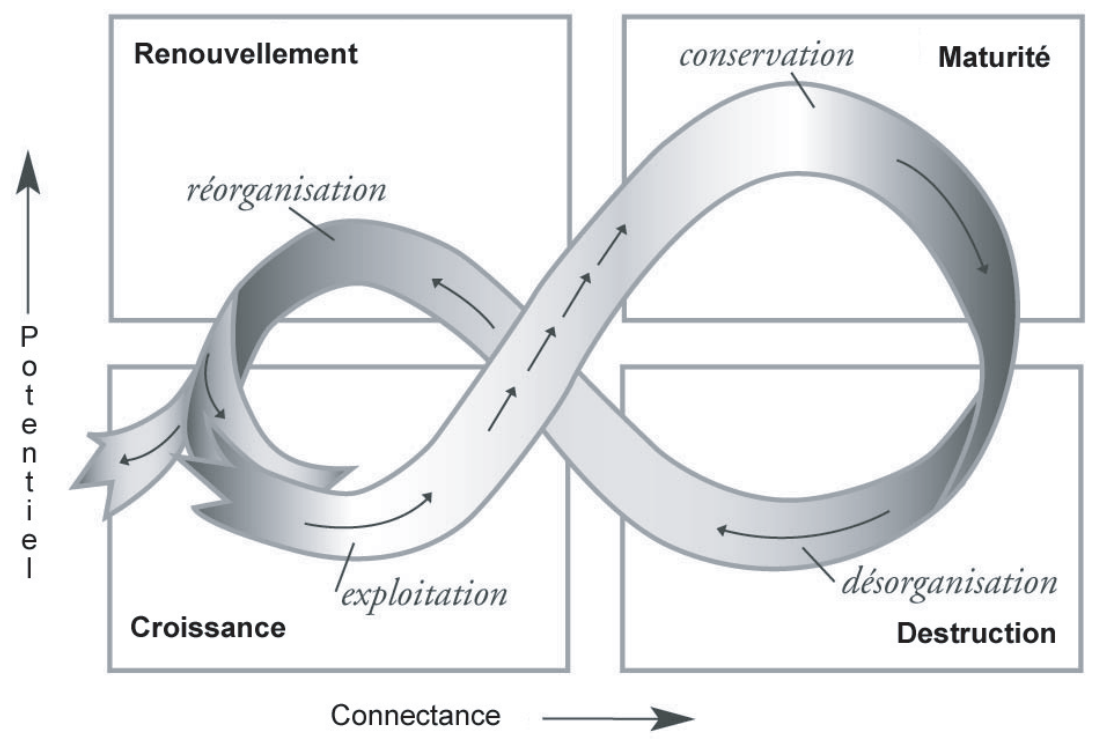

Figure 2.4

Représentation du cycle adaptatif d'un écosystème dont la trajectoire passe d'une phase d'exploitation à une phase de conservation (d'un système en croissance à un système à maturité), puis d'une phase de désorganisation à une phase de réorganisation (d'un système en destruction à un système en renouvellement). La transformation du système est d'abord lente (flèches courtes), puis rapide (flèches longues). En ordonnée, les ressources s'accumulent par accroissement de biomasse et de nutriments (potentiel). En abscisse, le degré $d^{\prime}$ organisation des variables qui contrôlent le système (connectance) augmente. La sortie du cycle, sur la gauche, figure un transfert vers un autre système (d'après Gunderson et Holling, 2002).

- le capital accumulé des systèmes considérés;

- leur connectance, ou lien entre leurs processus de contrôle interne ;

- leur résilience, ou aptitude à s'adapter à des perturbations, y compris soudaines et imprévues.

Dans un lac, par exemple, on assiste à une accumulation du capital de biomasse végétale, en même temps que, avec la connectance, le contrôle interne exercé par l'écosystème devient plus complexe. Le système perd alors de sa souplesse et devient plus rigide, vulnérable face aux crises. Ainsi, tout cycle adaptatif se construit par une alternance entre deux périodes qui s'opposent et se succèdent : I'une de croissance et de conservation, l'autre de destruction et de renouvellement. Quant à la résilience, la capacité d'adaptation à des surprises, elle accompagne ces deux périodes par une sorte de respiration : elle se contracte lors de la lente progression de la phase d'exploitation vers celle de conservation; elle s'épanouit lors de l'accélération qui conduit de la phase de désorganisation vers celle de réorganisation. Autrement dit, minimale 
à maturité, quand le système est le plus rigide, la résilience devient maximale au renouvellement du système, quand il est le plus inventif.

L'idée de cycle adaptatif a été associée à celle de l'emboîtement des échelles pour donner naissance à la métaphore de la «panarchie » (figure 2.5). Par emboîtement des échelles on entend une organisation en poupées russes dans laquelle un système $d^{\prime}$ 'ordre élevé comprend des sous-systèmes qui comprennent eux-mêmes $d$ 'autres sous-systèmes, et ainsi de suite. Il en est ainsi du bassin versant $d$ 'une rivière et de ses différents sous-systèmes jusqu'au microhabitat présent dans les interstices d'un banc de galets. Cette organisation impose de prendre en compte l'ensemble de ces échelles pour comprendre les causes et les conséquences de l'état d'une masse d'eau, et en prévoir les évolutions.

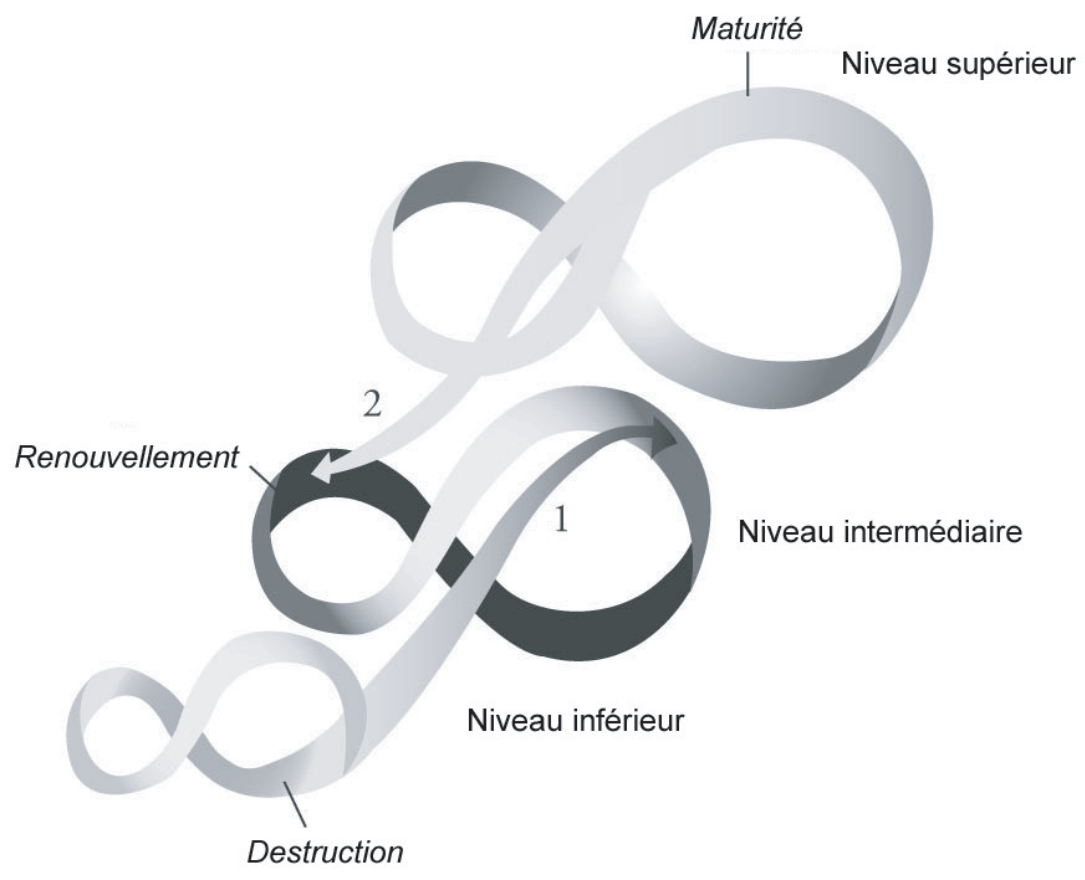

Figure 2.5

Organisation hiérarchique des systèmes écologiques. En (1), la phase de destruction du niveau d'échelle inférieur, plus petit et plus rapide, se répercute sur la phase vulnérable du niveau intermédiaire (par exemple, le déversement d'un produit toxique dans une roselière contamine l'ensemble d'un écosystème lacustre). En (2), le renouvellement de ce même niveau intermédiaire subit l'influence du potentiel accumulé et stocké dans le niveau d'échelle supérieur, plus grand et plus lent (par exemple, un massif forestier modère les variations de température des ruisseaux qui le drainent) (d'après Gunderson et Holling, 2002).

L'état des eaux continentales change donc sans cesse, tantôt progressivement, tantôt soudainement. II change au sein de configurations ressenties les unes comme désirables, les autres comme indésirables. Et l'aptitude d'un système écologique à se maintenir dans une de ces configurations dépend de sa 
résilience. Or, cette résilience change également : au fil des phases que parcourt chaque niveau d'échelle d'un système écologique, au fil des connexions possibles d'un niveau d'échelle à l'autre, de haut en bas, comme de bas en haut. Et donc, tout état d'une masse d'eau s'inscrit dans une dynamique de systèmes écologiques emboîtés, une dynamique qu'il faut prendre en compte pour orienter un système donné vers une configuration désirable ou, à l'inverse, pour l'écarter d'une configuration indésirable.

\subsection{Notion de socio-écosystème}

Les sociétés humaines utilisent les écosystèmes aquatiques et, par suite, les modifient, localement et globalement. En retour, elles sont capables d'ajuster leurs usages aux modifications qu'elles perçoivent. Cette interaction dynamique caractérise ce qu'il est convenu d'appeler des socio-écosystèmes (Walker et al., 2002). La résilience de ces socio-écosystèmes passe, notamment, par l'aptitude des sociétés humaines à tirer les leçons d'expériences passées pour anticiper et élaborer des plans pour l'avenir. Concernant les eaux continentales, cette aptitude conduit à des interrogations :

- Comment tel groupe social réagit-il aux changements du milieu naturel qu'il utilise?

- Comment parvenir à une utilisation équitable et durable des milieux naturels?

- Quelles structures sociales favorisent l'émergence de solutions à la dégradation des milieux naturels?

Ces questions relèvent des sciences de l'homme et de la société, et le grand défi posé à la gestion des socio-écosystèmes est justement d'articuler ces sciences avec celles de la nature, dans un souci de partage avec les gestionnaires et les décideurs de politiques publiques. Une utilisation durable des eaux continentales suppose en effet $d$ 'intégrer les dynamiques des écosystèmes aquatiques et celles des structures sociales qui relient les utilisateurs de ces écosystèmes.

En quel état de santé souhaitons-nous maintenir telle ou telle rivière? Une certaine dégradation est-elle acceptable? Si oui, que doit-on sacrifier? Si non, quel niveau de restauration viser? Les réponses à ces questions dépendent des objectifs qu'une société fixe à l'usage de ses rivières, mais aussi de critères choisis pour définir divers états possibles. L'approche proposée par la directive cadre européenne s'appuie sur des critères qui permettent de classer les cours d'eav et 
autres pièces d'eau en référence à des conditions "naturelles 》 ou "non perturbées ». Elle permet de fixer des standards aux projets d'aménagement, et ouvre ainsi un champ nouveau de collaboration aux scientifiques et aux gestionnaires.

\section{Gérer la résilience dans un environnement fluctuant}

Les biens et les services délivrés par les eaux continentales dépendent de variables à évolution lente, sujettes à des changements soudains. Ainsi, l'état d'un lac dépend des quantités de phosphore présentes dans le sol du bassin versant et dans les sédiments lacustres. Tant que ces quantités ne dépassent pas un seuil critique, la teneur de l'eau en phosphore est contrôlée par des processus d'assimilation et de décomposition, et le lac reste dans une configuration qui permet d'utiliser ses services. Le dépassement du seuil critique entraîne un changement de configuration : le système se retrouve placé dans une nouvelle dynamique qui ne permet plus d'utiliser ses services. Du point de vue de la gestion, il importe d'identifier les variables qui, à l'instar du phosphore, peuvent conduire au dépassement de seuils critiques.

L'identification de ces variables ne peut se concevoir sans référence à la notion d'échelle (§ 3.1). Parmi ces variables, la biodiversité apparaît comme une condition fondamentale de la résilience des socio-écosystèmes aquatiques (§ 3.2), d'autant plus que la mondialisation suscite des événements d'ampleur inédite, comme les invasions d'espèces exotiques (§ 3.3). Gérer cette résilience exige une réflexion sur les avenirs possibles des écosystèmes aquatiques (§ 3.4 ).

\subsection{De l'importance des échelles d'espace et de temps}

Les eaux continentales peuvent être perçues à plusieurs niveaux d'échelles, dans l'espace et dans le temps. Elles peuvent aussi être perçues à divers niveaux d'organisation biologique - individus, populations, communautés, écosystèmes. Aucun de ces niveaux n'est a priori meilleur que les autres, mais à chaque niveau correspondent certaines questions, certains outils et méthodes, et certaines conclusions possibles.

Ainsi, pour les facteurs de la distribution des végétaux fixés dans un lac: l'échelle continentale privilégie le rôle des facteurs climatiques (irradiance annuelle, température moyenne de l'air, longueur de la saison de croissance), l'échelle régionale privilégie le rôle des facteurs géomorphologiques (chimie des eaux, pentes des versants), l'échelle locale du lac privilégie la profondeur de 
l'eau, substitut de la pénétration de la lumière reçue... Les causes de la distribution de ces végétaux dépendent ainsi de l'échelle considérée, et une interprétation valable à une échelle pourra s'avérer inexacte à une autre. La difficulté est d'associer tout en les distinguant les différents niveaux d'échelles considérés, et de communiquer les résultats obtenus explicitement dans un contexte d'échelle. Car la confusion entre échelles est souvent source de malentendus, interdisant de parvenir à des réponses opérationnelles aux questions posées.

Concrètement, la relation entre échelles se pose en ces termes : dans quelle mesure une modification locale d'un écosystème aquatique peut-elle influencer un niveau régional, voire continental ? Autrement dit, le dysfonctionnement $d^{\prime}$ 'un écosystème aquatique est-il susceptible $d^{\prime}$ influencer de plus vastes niveaux d'échelles? Il semble possible de répondre affirmativement à cette question. Dans le domaine des eaux courantes, des impacts locaux peuvent se répercuter à des milliers de kilomètres, en d'autres milieux. Ainsi, les barrages du Mississippi et de ses affluents, en réduisant les débits du fleuve, ont annulé sa capacité de transport de nutriments, ce qui provoque le déclin des stocks de poissons et de crustacés dans le golfe du Mexique, et des effets semblables peuvent être prévus à propos du barrage des Trois Gorges sur la mer de Chine. Dans le domaine des eaux stagnantes, la décomposition de la biomasse associée aux réservoirs hydroélectriques peu profonds des climats chauds rejetterait des quantités de gaz carbonique telles que ces réservoirs pourraient être responsables de près de $15 \%$ des émissions de gaz à effet de serre, étant donné les projets de construction en cours.

\subsection{La biodiversité, condition de la résilience}

La biodiversité joue un rôle majeur dans le maintien de la résilience des écosystèmes. Ce rôle tient à l'existence de groupes fonctionnels, c'est-à-dire d'ensembles d'espèces aux fonctions écologiques similaires. Par exemple, parmi les invertébrés herbivores du fond d'un cours d'eau, certains broutent les algues de la surface des pierres, d'autres filtrent celles qui dérivent avec le courant, d'autres déchiquettent les fevilles tombées depuis les rives, s'attaquent aux tiges des macrophytes, etc.

Après perturbation, un écosystème se réorganisera d'autant mieux qu'il renferme divers groupes fonctionnels et, au sein de chacun de ces groupes, diverses espèces qui, tout en accomplissant une même fonction, répondent différemment aux perturbations. Ainsi, dans un lac, la résilience de la production primaire aux variations de température et d'éclairement dépend de la présence d'un nombre élevé d'espèces d'algues qui, parce qu'elles répondent différemment aux diverses combinaisons de température et d'éclairement, se remplacent au fil des saisons et maintiennent une production végétale pendant une grande partie 
de l'année. La diversité représente donc une sorte d'assurance face au risque (Loreau et al., 2001), et son érosion peut rendre plus vulnérables certaines fonctions des écosystèmes. Dans une situation d'incertitude, une gestion écologique des milieux aquatiques s'attachera à maintenir les groupes fonctionnels critiques - ceux qui comprennent peu d'espèces. La conservation de la biodiversité ne relève donc pas seulement de raisons éthiques, esthétiques ou psychologiques; elle relève aussi de raisons pragmatiques.

Or, le nombre des espèces vivant en eaux continentales diminue d'une manière préoccupante. Globalement, au moins 34 espèces de poissons $(7$ depuis 1970) ont disparu au cours du XX $X^{\mathrm{e}}$ siècle, et ce nombre doit être probablement doublé pour tenir compte des espèces disparues depuis le début du XIX siècle. Au niveau régional, de grandes extinctions ont frappé les eaux continentales, plus que d'autres milieux, au cours du XXe siècle. On estime ainsi à plus de $60 \%$ le taux de disparition des cichlidés endémiques dans le lac Victoria, suite à l'introduction de la perche du Nil, jointe à une pression de pêche, à une sédimentation et à une eutrophisation de plus en plus importantes (encadré 2.3). Aux États-Unis, le bassin versant de l'Alabama a vu disparaître 34 de ses 120 espèces de poissons avec la multiplication des barrages sur ses cours d'eau.

\title{
Encadré 2.3
}

\section{Les systèmes aquatiques africains : des modèles pour étudier l'évolution et les conséquences des changements climatiques}

\author{
Christian Lévêque, IRD et Muséum national d'histoire naturelle
}

Les systèmes aquatiques africains constituent $d^{\prime}$ 'excellents modèles pour appréhender le fonctionnement à long terme des écosystèmes et la dynamique de la biodiversité aquatique. Les situations écologiques y sont en effet très contrastées, depuis les lacs profonds dont l'existence est très ancienne jusqu'aux immenses plaines d'inondation associées aux grands fleuves, dont l'existence saisonnière dépend fortement des fluctuations climatiques.

Les Grands Lacs d'Afrique de l'Est (Tanganyika, Malawi) vieux de plusieurs millions d'années sont caractérisés par une faune hautement endémique de poissons et d'invertébrés. Ce sont de véritables laboratoires naturels pour l'étude des mécanismes de l'évolution et des processus de spéciation, où la co-évolution des espèces avec leur environnement a permis une grande diversification des réseaux trophiques. Ce sont également des observatoires privilégiés des conséquences des perturbations anthropiques: le fonctionnement écologique du lac Victoria a été profondément modifié 
par l'eutrophisation, la pêche excessive, les pollutions chimiques, et les introductions d'espèces, notamment celle la perche du Nil ou capitaine (Lates niloticus) et de la jacinthe d'eau (Eichhornia crassipes).

Les grands fleuves de l'Afrique nord-tropicale (Niger, Chari, Sénégal, et Nil) ont connu quant à eux des situations climatiques contrastées au cours de l'Holocène, avec des périodes très pluvieuses et d'autres marquées par la sécheresse. La composition de leur faune ichtyologique laisse penser que de nombreux échanges ont eu lieu entre les bassins lors des épisodes humides. Les vallées fossiles du Sahara sont les témoins de fleuves puissants qui alimentaient le lac Tchad depuis l'Ennedi et le Tibesti il y a quelques milliers d'années. Même au cours du siècle dernier, la superficie du lac Tchad $n^{\prime}$ a cessé d'évoluer en rapport avec les fluctuations climatiques, et le lac a parfois été réduit à un grand marécage. II ne comporte aucune espèce endémique, mais les peuplements ichtyologiques y sont composés en partie d'espèces adaptées aux conditions extrêmes, ce qui leur confère une certaine résilience quand on passe de conditions lacustres à des conditions palustres, et vice-versa.

Le fleuve Congo correspond à un des grands bassins fluviaux encore peu modifiés par l'homme : la densité de sa population y est restée relativement faible et aucun projet " pharaonique » n'y a encore été réalisé. De par son existence ancienne (bien avant le Miocène), il héberge une faune particulièrement riche, mal connue à cause de difficultés d'accès et menacée par un projet récurrent de détournement d'une partie des eaux du Congo vers le bassin du Tchad.

Les dynamiques à long terme des systèmes aquatiques africains et européens dépendent de facteurs différents. Les glaciations n'ont pas joué un rôle déterminant dans la mise en place de la biodiversité des systèmes aquatiques africains, mais les périodes de sécheresse peuvent avoir eu des effets dont on retrouve les traces dans la composition actuelle des peuplements (Lévêque et Paugy, 1999). Dans les deux cas, les espèces ont dû s'adapter à des changements de température et de régime des eaux. La comparaison de ces situations permet de mieux cerner les mécanismes biologiques mis en jeu par les espèces pour faire face aux changements de l'environnement. De nombreux peuplements aquatiques ont été l'objet de périodes successives d'extinction et de colonisation au cours desquelles le hasard et l'opportunité ont dû jouer un rôle essentiel. Ce qui conduit à un autre regard sur les introductions d'espèces : à long terme, ces peuplements paraissent capables d'intégrer de nouveaux immigrants. 
En 2000, plus de 3500 espèces de vertébrés et d'invertébrés liés aux eaux continentales étaient classées à haut risque d'extinction, et au moins $20 \%$ d'espèces de poissons d'eau douce menacées de disparition (UN/WWAP, 2003). Parmi les espèces emblématiques menacées figurent des espèces aussi diverses qu'un dauphin du Yang-tseu, un canard des rivières rapides d'Amérique du Sud, l'alligator chinois, une tortue de Thaïlande, l'axolotl du Mexique, l'esturgeon commun de France et de Géorgie. . Cette érosion de la biodiversité, souvent concomitante de destructions d'habitats, se développe sous l'influence des modes d'utilisation des terres, des invasions biologiques et du changement climatique, trois facteurs susceptibles d'agir différemment selon la latitude : les modes d'utilisation des terres surtout dans les régions tropicales, les invasions biologiques et les modes d'utilisation des terres en régions tempérées, le changement climatique en régions arctiques ou en haute altitude (Sala et al., 2000).

En fait, les changements climatiques récents ont déjà affecté beaucoup d'espèces continentales, terrestres et aquatiques. On observe déjà des déplacements d'aires de répartition vers les pôles de plus de $6 \mathrm{~km}$ par décennie en moyenne, de même qu'une plus grande précocité au printemps (Parmesan et Yohe, 2003). En outre, le réchauffement climatique agit en synergie avec d'autres facteurs, obligeant à adopter une perspective globale pour tenter d'enrayer l'érosion de la biodiversité des milieux aquatiques. Il faut par exemple tenir compte des déforestations dont dépend le sort de la faune et de la flore de l'Amazone, du Congo et de maints fleuves d'Asie du Sud-Est, soit près de la moitié des espèces de poissons d'eau douce de la planète. II faut aussi tenir compte des détournements des eaux dans le cadre des grands projets d'irrigation, des barrages et des canalisations qui partout fragmentent les réseaux hydrographiques. Il faut enfin voir l'érosion de la biodiversité comme une conséquence de tout un ensemble d'effets sur la physiologie des organismes vivants, leur phénologie, leur distribution géographique et leur adaptabilité, effets qui impliquent des transformations de la structure et de la composition des communautés et de nouvelles interactions entre les espèces.

Comment enrayer l'érosion de la biodiversité des eaux continentales?

- En protégeant sans tarder les habitats aquatiques, au-delà des réserves et des parcs, dans les milieux de la "nature ordinaire".

- Et en conservant la résilience des socio-écosystèmes par une meilleure compréhension des liens entre : 1) le fonctionnement des écosystèmes et les facteurs environnementaux, 2) la société, la biodiversité et les services rendus par les écosystèmes aquatiques. 


\subsection{La question des espèces envahissantes}

Introduites hors de leur région d'origine, certaines espèces s'installent et prolifèrent au détriment des espèces en place. Ce phénomène n'est pas nouveau, ni dû exclusivement à l'homme, mais il a été considérablement amplifié par le développement des transports, des voyages et des échanges commerciaux au cours des dernières décennies. Et ses effets peuvent s'avérer dramatiques visà-vis de la santé publique, de l'économie et de l'environnement. Les invasions biologiques apparaissent aujourd'hui comme la deuxième cause d'érosion de la biodiversité, après la destruction des habitats naturels (Mack et al., 2000).

Pourquoi une espèce normalement discrète dans son milieu d'origine devientelle envahissante - souvent après un temps de latence de plusieurs années - quand introduite dans un nouveau milieu? Première raison possible, cette espèce trouve des conditions favorables de développement sans être freinée par ses ennemis habituels, parasites, pathogènes ou prédateurs. Elle consacre donc une part plus importante de ses ressources pour croître et se reproduire dans la mesure où elle $n^{\prime}$ a plus à se défendre de ces ennemis. Les espèces envahissantes seraient ainsi des espèces pouvant exprimer leur caractère prolifique. Mais le succès d'une invasion dépend de plusieurs autres facteurs : nombre $d^{\prime}$ individus introduits, fréquence des introductions, vulnérabilité des communautés d'accueil, conditions de transport, d'établissement, d'expansion.

Parmi les effets les plus spectaculaires des invasions par des espèces d'origine étrangère, on cite souvent l'obstruction de certaines voies navigables par des plantes comme la jacinthe d'eau ou la disparition déjà évoquée d'espèces endémiques dans le lac Victoria. De même, les rats musqués et les ragondins, introduits pour leurs fourrures, creusent les berges des cours d'eau et aggravent les effets des crues, notamment en Europe centrale et méridionale. Tout aussi marquantes pour l'écologie des cours d'eau sont les colonisations de leurs berges, par exemple en région méditerranéenne par le robinier faux acacia et l'érable américain, mais aussi par des arbustes et des herbacées comme l'ambroisie, le faux-indigo, le buddleia, la balsamine géante, les jussies, la renouée du Japon, les verges d'or. En Afrique du Sud, certaines introductions d'eucalyptus et d'acacias ont été jusqu'à assécher des rivières dans la province du Cap.

Il n'est pas facile de lutter contre de telles invasions. Comment en effet identifier les attributs des espèces potentiellement envahissantes et comment prévoir les sites susceptibles d'être envahis? L'éradication est rarement possible une fois une espèce indésirable installée et son contrôle demande des efforts longs et coûteux. En 2002, la Convention pour la diversité biologique attirait l'attention sur les priorités à respecter : $d$ 'abord, éviter les introductions, sinon éradiquer précocement, et quand cela n'a pas été possible, exercer un contrôle à long 
terme. La mise en place de stratégies efficaces passe par des progrès à accomplir dans la compréhension épidémiologique des invasions, dans l'évaluation de leurs coûts directs et indirects, dans une prise de conscience par le public et les responsables politiques de leur gravité.

Faut-il proscrire l'installation de toute espèce d'origine étrangère? La distinction entre natives et étrangères n'est pas tovjours aisée : des espèces introduites il y a plusieurs siècles sont aujourd'hui naturalisées, au même titre que celles installées lors de changements climatiques datant de l'holocène. En outre, toute espèce introduite n'est pas forcément malfaisante : certaines s'avèrent capables $d^{\prime}$ assurer des fonctions écologiques manquantes sans devenir envahissantes. La plus grande vigilance s'impose cependant vis-à-vis des espèces à expansion rapide, d'autant plus que le changement climatique favorise les proliférations hors des aires d'origine.

\subsection{Construire des scénarios d'avenir}

Quel que soit notre niveau de connaissance, il sera toujours difficile de prévoir l'avenir des écosystèmes aquatiques. D'abord, parce qu'il s'agit de systèmes complexes, dont la dynamique dépend de conditions souvent imprévisibles sur le long terme. Ensuite, parce que ces systèmes sont étroitement couplés aux systèmes sociaux et donc soumis à des interactions qui, pour la plupart, demeurent inconnues. Telle prévision à propos de la qualité des eaux d'un bassin pourra susciter, en retour, de nouveaux comportements, et se trouver finalement démentie. Toute décision de gestion porte donc sur des systèmes complexes, aux dynamiques incertaines à moyen et à long terme. Une erreur courante est justement de sous-estimer ces incertitudes, d'ignorer les à-coups et les surprises dans les dynamiques écologiques.

La méthode des scénarios vise à identifier quels sont les avenirs possibles de tel ou tel écosystème aquatique sous l'effet, par exemple, de conditions climatiques inhabituelles, d'augmentations de population, d'améliorations techniques... Elle permet de recentrer l'attention sur les discontinuités qui risquent d'accompagner toute tendance à moyen ou à long terme. Elle facilite également l'émergence d'hypothèses de travail à propos des changements à venir, en mettant en regard différents usages des écosystèmes avec leur dynamique biophysique. À cet égard, l'élaboration de scénarios par les différents utilisateurs $d^{\prime}$ 'un écosystème aquatique les amène à prendre conscience des conséquences possibles des décisions de gestion.

Comment améliorer notre aptitude à prévoir les dynamiques des systèmes aquatiques continentaux et, en conséquence, répondre aux changements à moyen terme des socio-écosystèmes? Une réponse consiste à élaborer différents 
scénarios possibles. Nourrir ces scénarios suppose l'existence d'observatoires pérennes, susceptibles d'associer étroitement les scientifiques, les décideurs et les gestionnaires.

\section{Prescriptions pour la santé écologique des eaux continentales}

Ces dernières années ont vu se développer des recherches qui permettent en principe d'améliorer la santé écologique des eaux continentales. Les prescriptions se réfèrent : à l'environnement terrestre des eaux continentales (§ 4.1 ), aux besoins en eau des organismes aquatiques et riverains (§ 4.2), à l'eutrophisation des lacs et des rivières ( $\$ 4.3$ ), à l'aménagement des zones humides et des ripisylves (§ 4.4). Toutes ont des implications socio-économiques fortes, illustrant le bien-fondé de l'assimilation des eaux continentales à des "socio-écosystèmes ".

\subsection{Prendre en compte l'environnement terrestre des eaux continentales}

Les eaux continentales s'inscrivent dans un environnement terrestre dont elles sont indissociables. Non seulement leur fonctionnement écologique et biogéochimique dépend de cet environnement, mais ce dernier peut subir de leur part un effet en retour déterminant. Cette réalité conduit à l'idée d'une gestion de l'eau coordonnée avec les projets de territoire, l'eau entretenant des relations étroites et complexes avec les écosystèmes terrestres (encadré 2.4). Elle conduit aussi à la notion d'hydro-écorégion proposée pour regrouper des écosystèmes aquatiques réagissant de façon similaire aux facteurs de l'environnement (Wasson et al., 2005). Les approches d'écologie du paysage, privilégiant les relations entre organisation de l'espace et fonctionnement écologique, prennent ici tout leur sens (Décamps, 1996).

Les recherches engagées en 1963 dans la forêt expérimentale de Hubbard Brook, New Hampshire, ont révélé la complexité, l'ampleur et la durée des conséquences des déboisements sur les cours d'eau (Likens et Bormann, 1995). Elles ont par ailleurs démontré l'intérêt des stations de terrain équipées pour observer quels étaient les effets des changements d'utilisation des terres sur les écoulements. Mais il reste encore à quantifier ces effets, à diverses échelles, en régions tropicales dont les surfaces forestières diminuent, comme en Europe où elles ne cessent d'augmenter depuis deux siècles. En France, par exemple, cette surface dépasse actuellement le quart du territoire métropolitain, soit presque deux fois plus qu'en 1800, avec des conséquences à préciser sur les températures des eaux de surface, les transports des sédiments, les charges en nutriments... 


\title{
Encadré 2.4
}

\section{Eaux et territoires : enjeux}

\author{
Daniel Terrasson, Cemagref
}

Les communautés humaines se sont développées en fonction des ressources qu'elles pouvaient exploiter. L'eau a ainsi joué un rôle structurant majeur : pas d'implantation un tant soit peu pérenne sans une source d'eau potable, pas de gibier, d'animaux domestiques qui ne puissent s'abreuver. Les civilisations avançant, l'humanité a progressivement appris à exploiter le pouvoir de dilution de l'eau, sa force hydraulique, ses propriétés caloportrices. Les réseaux de transports se sont localisés autour des voies d'eau : plus grande facilité du transport fluvial, sites de vallées plus propices à l'établissement des voies de communication... L'artisanat puis l'industrie y ont puisé l'énergie, les eaux de lavage, de refroidissement. Même si les progrès de la chimie et de la technologie ont permis de découvrir des produits de substitution, l'eau n'en a pas moins gardé un rôle majeur y compris dans certaines industries de pointe comme par exemple les centrales nucléaires. Le simple examen d'une carte de France montre combien le développement s'est structuré en fonction du réseau hydrographique.

L'eau a également contribué à structurer l'occupation de l'espace chaque fois qu'elle pouvait constituer directement (submersions plus ou moins occasionnelles, zones désertiques) ou indirectement (insalubrité des zones humides) un facteur limitant. Très tôt, les sociétés ont cherché à se soustraire à ces contraintes de localisation, à l'excès ou à la pénurie d'eau. L'eau a eu en particulier de très fortes implications dans la culture et la technologie urbaine, ce dont témoignent les travaux archéologique dans la vallée de I'Indus (-2400 av. J.-C.). De nombreux développements régionaux n'ont été possibles que grâce à la construction d'aqueducs romains, au percement de canaux de liaison entre réseaux hydrographiques, à l'assèchement de marais, à la mise hors d'eau de vastes polders, au forage dans les nappes profondes, à la construction d'usines de dessalement d'eau de mer. L'ampleur de ces transformations a pu conduire, encore de nos jours, à des confrontations entre États.

Cependant, depuis quelques décennies, les sociétés occidentales voient leurs économies se dématérialiser. L'agriculture, grosse consommatrice d'eau, perd son poids économique et social, y compris dans les territoires ruraux. Les moteurs du développement ne sont plus basés sur l'exploitation de ressources naturelles, mais sur le capital humain, les effets d'agglomérations, les nouvelles technologies de la communication et de l'information. L'Europe se structure autour de grands axes urbains, dont certains peuvent certes prolonger les principaux réseaux hydrographiques (axe Rhin-Rhône) mais 
en dépassant largement leur emprise. D'une façon générale, le territoire en tant qu'unité d'organisation de la société, tend à s'asseoir sur un espace de plus en plus étranger aux logiques naturelles, géomorphologiques ou écologiques. La confrontation eaux-territoires soulève alors deux types d'enjeux.

1) Au niveau politique, la prise de conscience est d'autant plus forte qu'il ne peut y avoir de gestion de l'eau sans coordonner celle-ci avec les projets de territoire. En France, la nécessaire solidarité entre usagers est certes bien ancrée dans les esprits depuis la création des agences financières de bassin devenues agences de l'eau. Mais ces dernières, institutions sectorielles, ne permettent pas la mise en débat des modes de développement, des choix de planification et $d$ 'aménagement du territoire, et de leurs conséquences à plus ou moins long terme sur la ressource en eau. La nouvelle directive cadre européenne sur l'eau ne règle pas cet aspect et, dans sa séance du 25 juin 2004, l'Académie de l'eau qualifiait le territoire de " grand absent » de cette directive. Ce point de vue n'est sans doute pas partagé par tous; il souligne cependant l'idée que, dans le champ de l'environnement notamment, nombre de politiques publiques actuelles portent sur des enjeux qualitatifs qui croisent l'organisation sectorielle d'une administration héritée d'une société du besoin. Le véritable enjeu de la pollution des nappes par l'agriculture en Bretagne n'est-il pas in fine celui du choix entre deux modes de développement: I'un axé sur la filière agricole, l'autre sur la valorisation des atouts environnementaux? Des coordinations s'avèrent de plus en plus nécessaires entre des niveaux imbriqués de responsabilité spatiale ef sectorielle à l'échelle du territoire.

2) Au niveau scientifique, il s'agit de comprendre les interactions multiples entre une dynamique économique et sociale et les caractéristiques quantitatives et qualitatives d'une ressource naturelle. Cela pose notamment des questions fortes en termes d'emboîtement d'échelles spatiales (géographiques/hydrologiques/sociologiques/politiques) et temporelles dans un contexte d'incertitude sur l'évolution des politiques économiques et du climat. L'eau n'est pas seulement une ressource, c'est aussi la composante $d^{\prime}$ 'un milieu naturel qui entretient des relations complexes avec les écosystèmes terrestres. Sur un plan opérationnel, cela suppose non seulement des approches interdisciplinaires, mais aussi le décloisonnement de communautés scientifiques isolées dans leurs thématiques et qui ne partagent pas les mêmes concepts.

Globalement, l'irrigation agricole soustrait les plus grandes quantités d'eau aux systèmes aquatiques continentaux. Elle est responsable, les besoins des élevages compris, de plus de $70 \%$ des extractions d'eau du milieu naturel 
et conduit, suivant les bassins, à des pertes d'eau, des pollutions chimiques, la salinité des terres, ou l'épuisement des aquifères souterrains. Autre aspect de l'utilisation des terres, l'urbanisation croissante affecte elle aussi lourdement les écosystèmes aquatiques continentaux, particulièrement quand l'intégrité des zones d'interface n'est pas respectée, par exemple entre les eaux de surface et les eaux souterraines (encadré 2.2).

Les ripisylves jouent un rôle d'interface qu'il convient aussi de mieux apprécier (Naiman et al., 2005). Forêts (sylva) des rives (ripa), elles concernent des linéaires importants le long des réseaux hydrographiques et s'étalent plus ou moins largement de part et d'autre des cours d'eau : tantôt en simples boisements de berges au voisinage des lits mineurs, tantôt en véritables forêts alluviales complexes et diversifiées. Leur existence dépend de la présence d'une nappe phréatique peu profonde et d'inondations périodiques. Leur aptitude à favoriser la conservation de la biodiversité de la faune des cours d'eau est clairement établie, en zone agricole comme en zone urbaine. Elles correspondent certainement à des éléments clés des paysages aquatiques, indispensables à prendre en compte dans les opérations de conservation et de restauration.

La forêt permet-elle de gérer les ressources en eau? Cette question se pose alors que la forêt se déploie en France. À côté d'avantages démontrés (réduction des charges en sédiments), d'autres n'ont été vérifiés que sur de petits bassins versants. Déceler les tendances, notamment à l'échelle des grands bassins, demande des observations à long terme. Et toute généralisation se heurte à la variabilité des débits d'une forêt à l'autre, en fonction du sol, de la topographie, du climat et des pratiques forestières (Lavabre et Andréassian, 2000). Quantifier l'impact de la forêt sur le cycle de l'eau reste la condition nécessaire d'une gestion intégrée des eaux et des forêts.

\subsection{Prendre en compte les besoins des organismes aquatiques et riverains}

Au fil du temps, les organismes aquatiques et riverains se sont adaptés à la variabilité naturelle des régimes hydrologiques de leurs régions biogéographiques. Leurs cycles de vie se déroulent en synchronie avec les crues et les étiages. Or, parfois d'une manière spectaculaire, les activités humaines ont transformé la variabilité naturelle des régimes hydrologiques, soumettant les organismes aquatiques et riverains à de nouvelles conditions. Ces derniers ne survivent pas tous, et leur capacité de survie dépend des stratégies adoptées pour s'adapter aux régimes auxquels ils étaient habitués (tableau 2.4). 


\section{Adaptation}

\section{Organismes}

Traits d'histoire de vie

Dissémination des graines lors

de la décrue de printemps

Rapide allongement des racines

après germination

Stade de diapause lors de la saison

sèche

Éclosion retardée des œufs

d'une partie de la ponte

Frai ou émergence des alevins en

Saules et peupliers

Saules et peupliers

Plécoptères et autres insectes aquatiques

Éphémères

Poissons salmonidés période de faible probabilité de crue

Comportement

Déplacement vers des lieux abrités

Divers invertébrés aquatiques lors des crues

OEufs profondément déposés

Poissons salmonidés

dans le substrat

\section{Morphologie}

Enracinement important

Rejets de souches nombreux

Forme aplatie

Macrophytes aquatiques

Saules et peupliers

Divers invertébrés aquatiques

\section{Tableau 2.4}

Trois modalités d'adaptation des organismes d'eau courante aux crues et aux étiages (d'après Lytle et Poff, 2004).

Ces stratégies doivent être prises en compte pour prévoir les réactions des espèces aux modifications des régimes hydrologiques, tant en ce qui concerne l'amplitude de ces modifications que leur fréquence, leur durée, leur occurrence saisonnière, leur soudaineté (Poff et al., 1997). Il est encore nécessaire de prendre en compte la prévisibilité des événements hydrologiques. Soit par exemple des crues fréquentes et de grande ampleur : si ces crues sont 
prévisibles, la sélection favorise les espèces dont le cycle est synchronisé de manière à leur permettre de les éviter; si elles sont imprévisibles, elles n'ont qu'un faible pouvoir de sélection sur le calage du cycle de vie, même si elles causent de fortes mortalités. L'espèce survit alors en évitant « de mettre tous ses œufs dans le même panier ", à l'exemple de certains insectes aquatiques qui, par éclosions asynchrones décalées dans le temps, ont une chance d'éviter une éventuelle crue trop sévère. II faut ajouter que la durée de vie d'un organisme peut aussi jouer un rôle considérable : le long d'une même rivière de montagne, un saule subira de nombreuses crues de fonte des neiges au cours de sa vie, contrairement à certains insectes aquatiques dont le développement n'excède pas quelques mois.

Ainsi les organismes aquatiques et riverains ne sont pas tous égaux devant les modifications des régimes des débits. Certains ont acquis des traits vitaux qui leur permettent d'éviter ou même d'exploiter certaines crues ou étiages sévères; $c^{\prime}$ est le cas de nombreuses espèces $d^{\prime}$ 'insectes aquatiques aux stades adultes aériens ou des poissons migrateurs. Leurs cycles de vie sont adaptés aux régimes des débits en place, et ces organismes seront particulièrement vulnérables aux gestions qui déplacent les événements hydrologiques d'une période de l'année à une autre. D'autres organismes présentent plutôt des adaptations comportementales ou morphologiques. Ils résistent alors aux débits extrêmes au cas par cas, par exemple en s'enfonçant dans le substrat. Ces adaptations sont déclenchées par des variations de débit elles-mêmes et permettent de mieux résister aux altérations des régimes hydrologiques.

Quelle part des débits réserver à la santé des rivières? L'idée de réserver une part de l'eau au fonctionnement écologique des rivières est récente. Inscrite au National Water Act d'Afrique du Sud en 1998, elle a été généralisée par I'UICN : "Respecter les valeurs intrinsèques des écosystèmes, et les bénéfices qu'ils délivrent, implique de laisser de l'eau aux écosystèmes pour assurer leur fonctionnement. Cette eau, avec celle nécessaire à la satisfaction des besoins humains de base, constitue une réserve prioritaire sur tous les autres usages. " L'identification des débits ainsi à réserver ouvre la voie à une nouvelle éthique de l'utilisation de l'eau des rivières, les écosystèmes devenant des consommateurs légitimes de cette eau.

\subsection{Préserver les lacs et les rivières de l'eutrophisation}

Il a fallu près d'un demi-siècle de recherches, une concertation internationale, et des observatoires bénéficiant de moyens pérennes, pour comprendre les bases scientifiques de l'eutrophisation des eaux continentales. Comme celui de tout écosystème, le comportement des lacs et des rivières s'inscrit dans 
la durée, et son observation demande du temps. L'histoire des recherches sur l'eutrophisation des lacs est, à cet égard, révélatrice.

Dès les années 1950, les scientifiques s'intéressèrent aux facteurs qui déterminent la biomasse du phytoplancton des lacs. Leurs efforts, menés en de nombreux pays, aboutirent à une corrélation entre la biomasse de phytoplancton présente dans un lac et le phosphore apporté à ce lac. II devint alors possible d'évaluer l'état trophique d'un lac en s'appuyant sur le taux d'apport en phosphore, la profondeur moyenne du lac et le temps de rétention hydraulique de ce lac. Restait à prouver qu'on pouvait modifier cet état trophique en manipulant les apports de phosphore, ce que démontrèrent des expériences in situ, sur une série de lacs canadiens. Cependant, une dispersion considérable caractérisait encore les diagrammes représentant les biomasses de phytoplancton des lacs en fonction de leur charge en phosphore. Cette dispersion ne fut réduite que par la prise en compte de la structure des réseaux trophiques, notamment avec I'influence du zooplancton sur le métabolisme des nutriments (Schindler et al., 1990) et les effets en cascade des grands poissons carnivores jusqu'au phytoplancton (Carpenter et al., 2001). Enfin, pour affiner les prévisions, il fallut encore préciser tout un ensemble d'interactions au sein des communautés aquatiques, avec des sevils, des pseudo-équilibres multiples, des processus emmêlés.

Sans doute savons-nous mieux gérer la biomasse végétale présente dans un lac : en modifiant les apports de phosphore, la structure du réseau trophique, ou les deux. Sans doute a-t-il été possible - il est vrai non sans mal - d'utiliser les résultats obtenus pour bannir l'usage des détergents phosphatés, pour atténuer les effets des rejets industriels et municipaux, pour « sauver » certains lacs comme à Annecy ou au Bourget. Mais les connaissances acquises sont toujours difficiles à appliquer, tant les causes de l'eutrophisation des eaux sont variées, les territoires concernés étendus, et les implications sociologiques, économiques et politiques nombreuses. Préserver les lacs et les rivières de l'eutrophisation demande à la fois des observations à long terme, des recherches interdisciplinaires et une meilleure communication entre scientifiques, gestionnaires et décideurs des politiques publiques (encadré 2.5).

Peut-on prévoir les floraisons de cyanobactéries toxiques? Nombre d'entre elles sont susceptibles d'empoisonner les pièces d'eau à la faveur de processus d'eutrophisation, avec des conséquences sérieuses aux plans de l'économie et de la santé humaine. Or, il s'avère difficile de discriminer les espèces toxiques au microscope. Les méthodes de biologie moléculaire représentent une voie d'avenir car elles permettent l'identification rapide des gènes impliqués dans la biosynthèse des toxines, la détection des espèces potentiellement toxiques, et l'identification des conditions propices à la production de toxines. 


\title{
Encadré 2.5
}

\section{Changements récents dans l'écosystème lémanique}

\author{
Daniel Gerdeaux, Inra, Thonon
}

Les scientifiques ont perçu la menace d'eutrophisation du Léman dès la fin des années 1950, et lancé un programme pour suivre la qualité des eaux. Cette initiative a été reprise par les deux États suisse et français qui créèrent en 1962 la Commission internationale de protection des eaux du Léman, $\mathrm{CIPEL}^{6}$. Les principaux paramètres physicochimiques et la dynamique du plancton sont suivis une fois par mois a minima depuis plus de 40 ans, et des statistiques de pêche sont disponibles depuis cette date.

La concentration en phosphore des eaux du lac est passée de $10 \mu \mathrm{g} / \mathrm{l}$ en 1955 à $80 \mu \mathrm{g} / \mathrm{l}$ au début des années 1970. Les premières stations d'épuration ont alors été équipées pour la déphosphatation des eaux rejetées. La concentration en phosphore a continué d'augmenter jusqu'en 1980, atteignant alors $90 \mu \mathrm{g} / \mathrm{l}$. Depuis, particulièrement avec l'arrêt de l'emploi des phosphates dans les lessives textiles en Suisse en 1986, cette concentration s'est abaissée pour atteindre aujourd'hui $32 \mu \mathrm{g} / \mathrm{l}$. Toutes les stations d'épuration pratiquent désormais la déphosphatation et plus de $95 \%$ de la pollution domestique est raccordée à ces stations. Cet important effort d'épuration ne permet pas toutefois un retour à la concentration en phosphore des années 1950 et l'existence d'une charge résiduelle a conduit la CIPEL à se fixer l'objectif réaliste de $20 \mu \mathrm{g} / \mathrm{I}$ pour éviter les nuisances dues à l'eutrophisation.

Les nuisances les plus perceptibles concernent la transparence des eaux et la pêche. La prolifération du phytoplancton diminue la transparence de l'eau et la matière organique en excès sédimente, colmatant les fonds et consommant de l'oxygène lors de sa dégradation. Les invertébrés du fond les plus exigeants en oxygène disparaissent. Quant aux poissons, I'omble chevalier (Salvelinus alpinus) et le corégone (Coregonus lavaretus) voient leur survie embryonnaire fortement diminuer, voire s'annuler pour l'omble chevalier qui fraye très profond. Le tonnage pêché de ces deux espèces a été très bas lors du maximum de l'eutrophisation et, sans un soutien par déversement d'alevins, l'omble chevalier aurait pu disparaître comme en d'autres lacs eutrophes. La perche et les cyprinidés, qui se reproduisent au printemps en zone littorale, souffrent moins de l'eutrophisation, la perche devenant dominante avant de laisser la place aux cyprinidés, les seules espèces à supporter une forte eutrophisation. Ce processus est réversible, et le corégone est à nouveau l'espèce dominante au Léman. Outre la restauration de la qualité

${ }^{6}$ www.cipel.org 
de l'eau, l'alevinage et le réchauffement des eaux interviennent également dans ces changements.

En 30 ans, les eaux du fond du Léman ont subi un réchauffement de l'ordre de $1{ }^{\circ} \mathrm{C}$, et la stratification thermique annuelle a été avancée d'environ un mois. En surface, la dynamique saisonnière du phytoplancton suit ce décalage, et il en est de même de celle du zooplancton herbivore. Quant aux algues "automnales », elles se développent dès l'été dans les eaux profondes mais, pour la plupart filamenteuses, elles sont difficiles à consommer. Ces avancées dans la dynamique de la production modifient les rapports trophiques dans le lac, d'autant plus que les poissons réagissent différemment d'une espèce à l'autre. Les gardons, cyprinidés d'eau chaude, se reproduisent un mois plus tôt environ, contrairement aux perches, $d^{\prime}$ 'où un partage des ressources nutritives modifié, avec des conséquences sur les dynamiques des populations. Les corégones, à reproduction hivernale, voient leurs larves bénéficier d'eaux plus chaudes qu'il y a 30 ans et d'une ressource nutritive dont la dynamique est anticipée. Leur survie est probablement meilleure : les captures annuelles de cette espèce sont passées de moins de 50 tonnes dans les années 1970 à plus de 300 tonnes depuis 1997.

En outre, une pollution industrielle au mercure a frappé le Léman au début des années 1970 et, par contre-coup, la qualité de la chair des poissons pendant 10 ans. Les analyses actuelles révèlent des pesticides dont les concentrations cumulées demeurent inquiétantes.

\subsection{Aménager les zones humides et les ripisylves}

Les zones humides correspondent à des terrains, exploités ou non, habituellement inondés ou gorgés d'eau douce, salée ou saumâtre, de façon permanente ou temporaire. Quand elle s'y développe, la végétation y est dominée par des plantes hygrophiles pendant au moins une partie de l'année. Les zones humides n'occupent que 4 à $6 \%$ de la surface terrestre. Elles ont souvent été asséchées dans le passé, en raison de leur rôle dans les épidémies de malaria et autres maux, notamment dans les régions méditerranéennes. Depuis une trentaine d'années, la tendance est à les conserver, voire à les restaurer en régions tempérées, pour leur aptitude : 1) à stocker du carbone, 2) à maintenir la biodiversité, et 3) à éliminer certaines substances polluantes.

L'aptitude des zones humides à stocker du carbone tient à leur forte productivité végétale, jointe à des conditions d'anaérobiose favorables à l'incorporation 
dans le sol d'une matière organique végétale se décomposant lentement (Mitsch et Gosselink, 2000). Mais ce stockage peut s'inverser et les zones humides devenir des sources de carbone, rejetant du gaz carbonique et du méthane dans l'atmosphère, en raison de l'accélération de la décomposition végétale par une élévation des températures. Ces rejets sont d'ailleurs facilités par le fait que les végétaux des zones humides - Typha, Carex, Phragmites ou Juncus - se décomposent sur pied avant d'être incorporés dans les sédiments sous-jacents, et donc ont le temps de rejeter du carbone vers l'atmosphère. Les évaluations à cet égard méritent d'être confirmées, en climat subtropical comme en climat tempéré froid, notamment en Eurasie et en Amérique.

En France, les zones humides occupent 1,7 millions d'hectares, soit $3 \%$ du territoire national ; 50 espèces d'oiseaux en dépendent et $30 \%$ d'espèces végétales remarquables et menacées y sont inféodées. Ce sont des milieux fragiles (encadré 2.6), aux fonctions désormais reconnues : hydrologiques, biologiques, économiques, sociales et culturelles. Leur régression se poursuit de manière continue depuis le début du XX $X^{e}$ siècle. D'où le "Plan national d'action pour la reconquête des zones humides » élaboré en 1995. Sans doute convient-il, dix ans après son lancement, de dresser un bilan de ce plan. Mais il convient surtout de développer une véritable "ingénierie écologique » des zones humides pour purifier les eaux usées, produire de la biomasse, créer des habitats, ... à l'exemple des expériences qui se multiplient actuellement un peu partout dans le monde.

\section{Encadré 2.6}

\section{Le delta du Rhône : un milieu fragile}

Mireille Provansal, François Sabatier, Claude Vella, Grégoire Maillet, Cerege, UMR CNRS, universités Aix-Marseille I et III

Le delta du Rhône est un milieu fragile, menacé à la fois par le recul actuel du littoral, la montée du niveau marin et les inondations du fleuve. Il subit depuis deux siècles diverses transformations, en relation avec l'aménagement du fleuve et les activités agricoles, industrielles et touristiques.

Le delta est une construction sédimentaire récente. La progradation du trait de côte - 10 à $20 \mathrm{~km}$ depuis 6000 ans - tient au ralentissement de la montée du niveau marin et aux apports alluviaux du Rhône. La montée du niveau marin, d'abord rapide entre 18000 et $6000 \mathrm{BP}(10 \mathrm{~mm} / \mathrm{an}$ en moyenne), s'est ensuite progressivement ralentie, le 0 NGF étant atteint à la fin du premier millénaire ap. J.-C. Les apports du Rhône ont été abondants, en particulier à partir du Néolithique avec le début des défrichements 
et des mises en culture. Leurs variations ont induit plusieurs avancées rapides, déplacements et/ou multiplications des embouchures, stabilisations et reculs du trait de côte. La dernière avancée importante du delta est associée au Petit Âge Glaciaire, période de crise hydrologique et de démographie rurale génératrice d'érosion, donc d'apports sédimentaires abondants.

Le $X X^{e}$ siècle s'individualise par l'affinement granulométrique et la réduction quantitative des apports fluviaux (de 20 à 8-10 Mt environ), notamment suite à la réduction des surfaces agricoles, à la reforestation des Alpes, aux barrages hydroélectriques et aux dragages. Le littoral recule et prend une forme lobée, tout en bénéficiant encore d'un héritage sédimentaire, mais en cours de démantèlement et non renouvelable. Son " équilibre dynamique » est fragilisé par la montée du niveau marin et la réduction des apports du fleuve. Cette montée relative, mesurée au centre du delta, atteint $2 \mathrm{~mm} / \mathrm{an}$ depuis 1905, et le bilan sédimentaire du système littoral est devenu négatif depuis 50 ans, avec un déficit de $700000 \mathrm{~m}^{3} /$ an entre 0 et $-20 \mathrm{~m}$ et un recul quasi généralisé du linéaire côtier ( $4 \mathrm{~m} / \mathrm{an}$ en moyenne). La moitié sud du delta risque ainsi d'être submergée : $30 \%$ de sa surface est à une altitude inférieure à 0,50 mètres sous le niveau moyen de la mer, et le village des Saintes-Maries de la Mer pourrait être bientôt encerclé par la mer. En outre, la remontée de la nappe d'eau salée affecte les étangs méridionaux, mettant en péril des milieux à forte valeur écologique et, à plus long terme, la Camargue rizicole.

Ces effets s'ajoutent à une moindre fréquence des crues depuis la fin du $\mathrm{XIX}$ siècle, et au ralentissement des apports sédimentaires à l'embouchure. Le fleuve ne contribue plus que faiblement à l'alimentation littorale, avec un temps de rétention élevé des sables au sommet du prodelta. Quant aux inondations, elles sont localisées au niveau des brèches dans les digues insubmersibles de 1860, essentiellement sur le Petit Rhône. L'apport d'alluvions dans le delta dépend donc pour l'essentiel d'entrées d'eau liées à une irrigation qui, réalisée au printemps et en été à partir d'un fleuve peu chargé, ne permet plus l'exhaussement naturel de la plaine. En outre, les aménagements pour la navigation ont rétréci le chenal de plus de $400 \mathrm{~m}$ et l'ont approfondi de 1 à $4 \mathrm{~m}$, abaissant la ligne d'eau, ce qui favorise le drainage de la plaine, mais fragilise les digues.

En fait, le delta du Rhône subit depuis 150 ans une évolution, comparable à celle de la plupart des deltas méditerranéens, le fleuve ne lui apportant plus les sédiments dont il a besoin. Cependant, les crues récentes montrent que le Rhône est encore capable de transporter des charges solides abondantes, avec une fraction sableuse non négligeable. La question se pose donc d'améliorer les relations entre le bassin versant, le fleuve et le delta, jusqu'au trait de côte. Et les recherches doivent déterminer quelles menaces font peser une 
accélération de la vitesse de montée du niveau marin et de la fréquence des tempêtes.

La défense côtière a privilégié jusqu'ici les enrochements artificiels, avec des impacts paysagers et écologiques peu compatibles avec l'image de marque de la Camargue. Devant l'efficacité très variable de ces ouvrages s'impose la nécessité d'innover, voire d'accepter un recul raisonné du littoral du delta.

Les zones riveraines des cours d'eau jouent-elles un rôle de filtre vis-à-vis des pollutions diffuses? On l'admet pour l'azote, le phosphore et certains pesticides entraînés par ruissellement vers les cours d'eau des régions agricoles. Certains sites riverains jouent ce rôle, quand la production végétale y est forte, le temps de résidence de l'eau élevé, et les écoulements souterrains peu profonds. Mais il faut se garder de généraliser, et l'idée de restaurer la qualité des eaux des rivières grâce aux filtres riverains ne saurait être extrapolée sans réflexion à l'échelle d'un grand bassin versant, ni à toute pollution diffuse. Elle relève d'une ingénierie écologique des zones humides, prometteuse mais encore à développer.

\section{Un devoir de vision planétaire}

Plus d'un milliard d'hommes dans le monde boivent une eau contaminée et des pays entiers vivent des pénuries dramatiques pour leur développement agricole et industriel. Dans les plus pauvres d'entre eux sévit une mortalité prématurée par manque d'eau ou par maladies liées à l'eau. Et cette situation risque de perdurer, exacerbée par la croissance démographique, les pollutions et le changement climatique. La crise mondiale de l'eau impose aux scientifiques un devoir de vision planétaire : pour comprendre la dynamique d'un système - le système hydrologique mondial - dont dépend l'avenir de l'humanité (§ 5.1), pour dégager, dans toute leur complexité, les causes des disparités régionales (§ 5.2), pour aider à résoudre les problèmes, notamment par un bon usage de l'écologie (§ 5.3).

\subsection{Le système hydrologique global}

Le cycle de l'eau dépend de nombreux facteurs humains et non humains, tous interdépendants, au sein du "système hydrologique global 》. Les sociétés humaines dépendent de ce système, qu'elles contribuent à modifier de plus en plus. En outre, le système hydrologique global comprend deux autres composantes : I'une, physique, renvoie aux écoulements, à la géomorphologie, aux 
processus d'érosion et de sédimentation; l'autre, biologique et biogéochimique, renvoie aux organismes aquatiques et riverains, aux écosystèmes qui leur sont associés, à la biodiversité. À l'évidence, l'accroissement de la population mondiale (et les besoins liés à cet accroissement) transforme ce système. Il est urgent d'évaluer l'ampleur du changement qui affecte le système hydrologique global, ses causes, ses conséquences, ainsi que sa résilience (Framing Committee of the GWSP, 2004).

Le système hydrologique est en effet transformé à l'échelle de la planète, quantitativement avec les effets des barrages, les extractions d'eau ou les transferts, qualitativement avec les rejets ponctuels et diffus de substances polluantes diverses. Ainsi, $20 \%$ des 11000 espèces de poissons d'eau douce ont disparu ou ont été menacées de disparition au cours des dernières décennies (UN/WWAP, 2003). Et les peuplements présents ne correspondent plus aux mêmes assemblages, suite à la fragmentation du cours des rivières et à l'introduction d'espèces exotiques.

Le système hydrologique global est aussi caractérisé par sa connexité à l'échelle planétaire. Ces connexions se manifestent dans l'espace, des événements locaux ou régionaux se répercutant à des distances éloignées, avec parfois des effets en retour. Elles se manifestent aussi dans le temps, ces mêmes événements prolongeant leurs effets sur le long terme, avec des temps de latence entre causes et effets, et des héritages d'actions passées. La déforestation des plateaux chinois a ainsi multiplié par 10 la charge en sédiments du fleuve jaune, provoquant des transformations du lit du fleuve et favorisant des inondations à des centaines de kilomètres plus loin et des centaines d'années plus tard.

La connexité du système hydrologique oblige à repenser nos besoins d'information à une échelle mondiale (tableau 2.5). Ces besoins concernent par exemple la pollution des eaux continentales par voie atmosphérique, divers polluants volatils - composés organiques, mercure, nutriments - pouvant être transportés par l'air et contaminer des régions éloignées de leur lieu de production (Swackhamer et al., 2004). Certaines bioaccumulations sont particulièrement préoccupantes : celle des organochlorés parvenant aux régions les plus froides de la planète, étant donné la physiologie des organismes en place, la dynamique des chaînes trophiques, et la longueur des cycles vitaux, celle aussi du mercure et de ses effets sur la santé humaine.

Comment les dégradations observées à une échelle locale se répercutent-elles aux échelles plus globales? Comment se propagent-elles? Quelles sont les régions sensibles, les sevils critiques? Quels usages et quels besoins en pâtissent? Quelles sociétés humaines en souffrent le plus et quelles mesures prendre? Répondre à ces questions suppose la réunion de diverses perspectives, depuis celle 


\begin{tabular}{|c|c|c|}
\hline \multicolumn{3}{|c|}{$\begin{array}{l}\text { Forces de contrôle } \\
\text { - Climat } \\
\text { - Population } \\
\text { - Couvert des terres } \\
\text { - Extraction de l'eau } \\
\text { éveloppement économique } \\
\text { - Gouvernance }\end{array}$} \\
\hline \multicolumn{3}{|c|}{ Variables d'état } \\
\hline $\begin{array}{l}\text { Composantes } \\
\text { physiques }\end{array}$ & $\begin{array}{l}\text { Composantes } \\
\text { biogéochimiques }\end{array}$ & $\begin{array}{l}\text { Composantes } \\
\text { humaines }\end{array}$ \\
\hline $\begin{array}{l}\text { - Débit des rivières } \\
\text { - Régimes des débits } \\
\text { - Volumes de stockage }\end{array}$ & $\begin{array}{l}\text { - Richesse spécifique } \\
\text { - Habitat } \\
\text { - Qualité de l'eau }\end{array}$ & $\begin{array}{l}\text { - Accès à l'eau pure } \\
\text { - Occurrence } \\
\text { maladies liées à l'eau }\end{array}$ \\
\hline \multicolumn{3}{|c|}{ Facteurs de santé humaine } \\
\hline $\begin{array}{l}\text { Entre composantes } \\
\text { physiques et } \\
\text { humaines }\end{array}$ & $\begin{array}{l}\text { Entre composantes } \\
\text { physiques et } \\
\text { biogéochimiques }\end{array}$ & $\begin{array}{l}\text { Entre composantes } \\
\text { humaines et } \\
\text { biogéochimiques }\end{array}$ \\
\hline $\begin{array}{l}\text { - Disponibilité en eau } \\
\text { par personne } \\
\text { - Index de pauvreté en eau }\end{array}$ & $\begin{array}{l}\text { - Concentrations } \\
\text { en polluants }\end{array}$ & $\begin{array}{l}\text { - Qualité de l'eau } \\
\text { d'origine }\end{array}$ \\
\hline
\end{tabular}

Tableau 2.5

Les forces de contrôle (climat, population, ...) agissent sur les variables d'état du système hydrologique composantes physiques, biogéochimiques, humaines dont les interactions définissent des facteurs de santé humaine (d'après Framing Committee of the GWSP, 2004).

des sciences de la nature jusqu'à celles des sciences de l'homme et de la société, de manière à comprendre les dynamiques en cause.

\subsection{Disparités régionales}

Le problème du système hydrologique global se décompose en une multitude de problèmes locaux et régionaux, obligeant à rechercher des solutions 
pratiquement au cas par cas. Une vision planétaire doit rester attentive à ces défis régionaux. Le Global International Waters Assessment (GIWA), une évaluation des eaux internationales de la planète placée sous l'égide du Programme des Nations unies pour l'environnement (PNUE), distingue ainsi 66 régions dans son analyse des socio-écosystèmes aquatiques de la planète (Hempel et Daler, 2004). Ces régions illustrent divers aspects de la crise de l'eau dans le monde. Elles montrent aussi à quel point cette crise dépend de problèmes de gouvernance, et donc la nécessité de mieux prendre en compte la dimension humaine de l'évolution des socio-écosystèmes.

Le bassin du lac Tchad connaît ainsi une sécheresse persistante depuis une trentaine d'années. Une désertification aux conséquences dramatiques pour 44 millions d'habitants affecte lourdement l'ensemble des activités économiques de la région - pêche, agriculture, élevage - et induit des tensions, des exodes, des conflits. La Commission du bassin du lac Tchad, agence intergouvernementale comprenant le Tchad, le Niger, le Nigeria, le Cameroun, la République Centrafricaine, auxquels s'est ajouté le Soudan, est à l'origine d'un plan d'action pour « renverser les tendances à la dégradation des terres et des eaux " dans le socio-écosystème du bassin du lac Tchad. Cette dégradation renvoie en effet à des questions de démographie, d'économie, de manque de formation, d'absence de cadres juridiques et de gouvernance - toutes causes qui empêchent de mettre en place une stratégie de gestion intégrée, raisonnée et équitable des ressources en eau.

Un même besoin de gouvernance en matière de gestion des ressources en eau marque d'autres régions de la planète : baie du Bengale, bassins de la mer d'Aral, de la mer de Chine, etc. De nombreux pays partagent en effet des bassins hydrographiques avec un ou plusieurs autres pays - des bassins dans lesquels vit plus de $40 \%$ de la population mondiale, et dont dépendent les zones côtières qui devraient comprendre 75 \% de la population mondiale en 2030.

Comment remédier aux pénuries qui frappent de nombreux pays de la planète, comment enrayer les pollutions, les surexploitations des ressources vivantes, les destructions d'habitats? Comprendre les causes se révèle nécessaire mais insuffisant. Il faut encore s'engager dans des actions concrètes. D'où l'importance d'une participation active aux programmes internationaux qui, en Amérique du Sud, en Afrique, en Asie, mettent en place partenariats, réseaux et échanges pour tenter de relever les défis posés par la gestion des eaux continentales.

\subsection{Du bon usage de l'écologie}

Le devoir de vision planétaire s'accompagne d'un autre devoir face aux problèmes environnementaux - celui d'une approche pragmatique, réflexive, 
éloignée des idéologies. Dans une telle perspective, l'écologie des eaux continentales est indispensable à la prévision des trajectoires possibles suivies par les écosystèmes aquatiques après perturbations, naturelles ou anthropiques. Elle est aussi indispensable dans toute tentative visant à influencer ces trajectoires. À une condition cependant : être insérée dans des ensembles allant des sciences de la Terre à celles de l'homme et de la société.

Les scientifiques sont de plus en plus interrogés sur l'avenir des eaux continentales, qu'elles soient superficielles ou souterraines et qu'il s'agisse de lacs, de réservoirs, de rivières ou de zones humides. Répondre à cette attente suppose des recherches organisées d'une manière cohérente, interdisciplinaire et pérenne, suppose aussi de tirer tout le parti possible de nouvelles technologies - cartographie, télédétection, visualisation, traçage - qui permettent de détecter comme jamais les changements dans les écosystèmes, et de les suivre. Le principal défi est désormais de mettre un savoir foisonnant au service de prévisions toujours améliorables.

Dans le domaine des eaux continentales, le bon usage de l'écologie est donc indissociable d'une approche multidisciplinaire des problèmes, d'un décloisonnement des connaissances acquises et à acquérir, d'une liaison plus étroite entre la recherche et la gestion. Il est surtout indissociable d'une participation active aux programmes développés dans les pays en développement - ceux dans lesquels les problèmes de l'eau se poseront avec le plus d'acuité dans les décennies à venir.

\section{Conclusion}

Les changements planétaires en cours modifient profondément nos façons de concevoir le fonctionnement écologique des eaux continentales. De nouveaux débats bousculent nos connaissances; ils sont illustrés dans ce chapitre par 14 questions clés (paragraphes en italique) et 6 encadrés. Ces débats renouvellent les bases théoriques de la dynamique des écosystèmes et les pratiques de gestion. Nous devons par exemple tirer les conséquences des liaisons existant entre les milieux aquatiques et les territoires de leurs bassins versants, accepter l'idée que l'intégrité et la viabilité des écosystèmes aquatiques et riverains ne s'expriment que dans une dynamique de changements et de fluctuations, nous préoccuper de la capacité des socio-écosystèmes à s'adapter aux changements environnementaux à venir.

Cependant, en dépit des progrès accomplis dans la connaissance des écosystèmes aquatiques continentaux, ces derniers continuent à se dégrader, en 
France et dans le monde. Cette dégradation, quantitative et qualitative, est préoccupante car, inégalement répartie, elle est source d'inégalités et de conflits à venir. Or, la dégradation des milieux aquatiques continentaux n'est pas inéluctable, et les recommandations ci-après peuvent contribuer à renverser les tendances observées actuellement.

À la base de toute politique de développement durable, il est nécessaire de réserver - en quantité, en qualité et en temps voulu - la part de l'eau qui revient au fonctionnement des écosystèmes. Ces derniers sont en effet des utilisateurs légitimes de l'eau, et la part qui leur revient doit primer sur tout autre usage, une fois satisfaits les besoins humains de base. Ce fondement demande à être enseigné à tous niveaux, et l'éducation du public s'avère ici essentielle. II faut faire savoir que les lacs, les rivières, les zones humides, et les eaux souterraines qui les alimentent ne rendront les services que nous pouvons en attendre que s'ils sont en bon état de fonctionnement écologique. Il faut aussi faire savoir que ceci impose de se soucier de la résilience de ces écosystèmes face aux changements.

Il est aussi nécessaire d'organiser le partage des connaissances entre scientifiques, gestionnaires et décideurs. À cet égard, une structuration des recherches sur les écosystèmes aquatiques continentaux dans le cadre d'un observatoire de type ORE (observatoire de recherche sur l'environnement) ${ }^{7}$ est vivement recommandée. Cette structuration pourrait être pilotée en liaison avec un réseau de sites-ateliers et des observatoires opérationnels tels que les agences de l'eau. Un tel groupement permettrait de combler le vide laissé par la disparition de l'ancien GIP Hydrosystèmes et de renforcer une démarche scientifique commune ${ }^{8}$.

Cette structure améliorerait notamment la cohérence des actions de surveillance, de protection et de recherche dans le domaine des eaux continentales, avec :

- une surveillance de l'état de santé des écosystèmes aquatiques basée sur : des indicateurs biologiques correctement identifiés (systématique des espèces), des situations de référence acceptables (souhaits des parties prenantes), une grande vigilance à l'égard des invasions biologiques (traits biologiques des espèces susceptibles de devenir envahissantes) ;

\footnotetext{
7 http://www.recherche.gouv.fr/recherche/fns/ore.htm

${ }^{8}$ Contrairement à des craintes parfois exprimées, les observatoires de recherche tels que nous les concevons n'ont rien de refuges pour des travaux de suivi routinier. L'exemple du programme LTER (Long Term Ecological Research) montre qu'un dynamisme propice aux innovations et aux découvertes est au contraire favorisé par la durée, si cette dernière va de pair avec des évaluations rigoureuses.
} 
- une protection des habitats aquatiques, étape indispensable pour enrayer les pertes de biodiversité des lacs, rivières et zones humides et, par suite, pour développer la résilience des socio-écosystèmes liés à l'eau ;

- une recherche visant à :

- élaborer des scénarios à partir de sites-ateliers pérennes associant chercheurs, gestionnaires et décideurs,

- associer l'état des eaux continentales à l'utilisation des territoires de leurs bassins versants, notamment par l'usage des nouvelles méthodes d'observation de la Terre,

- identifier les conditions propres au développement d'espèces toxiques lors des phénomènes d'eutrophisation,

- développer une ingénierie des zones humides.

Enfin, il est inacceptable que notre communauté scientifique nationale soit pratiquement absente d'actions internationales aussi importantes pour l'avenir des eaux continentales de la planète que "l'Évaluation des écosystèmes pour le Millénaire » ou "l'Évaluation globale des eaux internationales " ${ }^{9}$, toutes deux lancées dans le cadre du Programme des Nations unies pour l'environnement (PNUE) : par exemple, la France ne figure pas parmi les 27 pays représentés dans le conseil de direction de la première de ces actions et ne participe pas à la seconde.

La vitalité de la recherche française dans le domaine des eaux continentales dépend d'une action forte pour assurer sa présence dans les programmes internationaux en cours et à venir.

\section{Références bibliographiques}

Balmford A. et al. (2002). Economic reasons for conserving wild nature. Science, 297 : 950-953.

Bellwood DR. et al. (2004). Confronting the coral reef crisis. Nature, 429 : 827-833.

Carpenter SR. et al. (2001). Trophic cascades, nutrients, and lake productivity : whole-lake experiments. Ecological Monographs, 71 : 163-186.

Commission européenne. Décision 2000/60/CE établissant un cadre pour une politique communautaire dans le domaine de l'eau. Journal Officiel, L 327 du $22 / 12 / 2000$.

\footnotetext{
${ }^{9}$ GIWA : Global International Waters Assessment (Hempel et Daler, 2004).
} 
Costanza R. et al. (1997). The value of the world's ecosystem services and natural capital. Nature, 387 : 254-260.

Décamps H. (1996). The renewal of floodplain forests along rivers : a landscape perspective. Verhandlungen der Internationalen Vereinigung für theoretische und angewandte Limnologie, 26 : 35-59.

Folke C. et al. (2002). Resilience and sustainable development : building adaptive capacity in a world of transformation. Ambio, 31 : 437-440.

Framing Committee of the GWSP (2004). The Global Water System Project : Science Framework and Implementation Activities. www.gwsp.org

Gibert J., Mathieu J., Fournier F. (eds.) (1997). Groundwater / Surface Water Ecotones: Biological and Hydrological Interactions and Management Options. Cambridge University Press.

Gunderson L., Holling CS. (eds.) (2002). Panarchy : Understanding Transformations in Human and Natural Systems. Island Press.

Hempel G., Daler D. (2004). Why a global international waters assessment (GIWA)? Ambio, 33 : 2-6.

Hering D. et al. (2004). Overview and application of the AQEM assessment system. Hydrobiologia, $516: 1-20$.

Kalff J. (2002). Limnology. Prentice-Hall. Upper Saddle River, New Jersey, USA.

Karr JR., Chu EW. (1999). Restoring life in running waters. Island Press.

Lavabre J., Andréassian V. (2000). Eaux et Forêts : la forêt, un outil de gestion des eaux? ECOFOR. Cemagref éditions.

Lévêque C., Paugy D. (eds.) (1999). Les poissons des eaux continentales africaines. Diversité, écologie, utilisation par l'homme. IRD, Paris.

Likens GE., Bormann FH. (1995). Biogeochemistry of a forested ecosystem. 2nd edition. Springer-Verlag, New York, USA.

Loreau M. et al. (2001). Biodiversity and ecosystem functioning : current knowledge and future challenges. Science, 294 : 804-808.

Lytle DA., Poff NL. (2004). Adaptation to natural flow regimes. Trends in Ecology and Evolution, 19 : 94-100.

Mack RN. et al. (2000). Biotic invasions : causes, epidemiology, global consequences, and control. Ecological Applications, 10 : 689-710.

Mitsch WJ., Gosselink JG. (2000). Wetlands. 3rd Edition. John Wiley and Sons, New-York, USA. 
Naiman RJ., Décamps H., McLain M. (2005). Riparia : Ecology, Conservation, and Management of Streamside Communities. Academic Press.

Palmer TN., Räisänen J. (2002). Quantifying the risk of extreme seasonal precipitation events in a changing climate. Nature 415 : 512-514

Parmesan C., Yohe G. (2003). A globally coherent fingerprint of climate change impacts across natural systems. Nature, 421 : 37-42.

Poff NL. et al. (1997). The natural flow regime : a paradigm for river conservation and restoration. BioScience, 47 : 769-784.

Postel SA., Richter B. (2003). Rivers for Life : Managing Water for People and Nature. Island Press.

Pourriot R., Meybeck M. (eds.) (1995). Limnologie générale, Masson, Paris.

Sala OE. et al. (2000). Global biodiversity scenarios for the year 2100. Science, 287 : 1770-1774.

Scheffer M. et al. (2001). Catastrophic shifts in ecosystems. Nature 413 : 591596.

Schindler DW. et al. (1990). Effects of climatic warming on lakes of the central boreal forest. Science 250 : 967-970.

Swackhamer DL. et al. (2004). Impacts of atmospheric pollutants on aquatic ecosystems. Issues in Ecology No 12. Ecological Society of America.

UN/WWAP (United Nations/World Water Assessment Programme) 2003. UN world water development report : water for people, water for life. UNESCO and Berghahn Books.

Walker B. et al. (2002). Resilience management in social-ecological systems : a working hypothesis for a participatory approach. Conservation Ecology, 6 : 14 (online) URL : http://www.consecol.org/vol6/iss 1/art14

Wasson JG. et al. (2004). Les hydro-écorégions : une approche fonctionnelle de la typologie des rivières pour la directive cadre européenne sur l'eau. Ingénieries, 40 : 3-10. 
